\title{
DENSE CORE FORMATION IN SUPERSONIC TURBULENT CONVERGING FLOWS
}

\author{
Hao Gong and Eve C. Ostriker \\ Department of Astronomy, University of Maryland, College Park, MD 20742-2421, USA; hgong@ astro.umd.edu, ostriker@astro.umd.edu \\ Received 2010 September 27; accepted 2011 January 12; published 2011 February 16
}

\begin{abstract}
We use numerical hydrodynamic simulations to investigate prestellar core formation in the dynamic environment of giant molecular clouds, focusing on planar post-shock layers produced by colliding turbulent flows. A key goal is to test how core evolution and properties depend on the velocity dispersion in the parent cloud; our simulation suite consists of 180 models with inflow Mach numbers $\mathcal{M} \equiv v / c_{s}=1.1-9$. At all Mach numbers, our models show that turbulence and self-gravity collect gas within post-shock regions into filaments at the same time as overdense areas within these filaments condense into cores. This morphology, together with the subsonic velocities we find inside cores, is similar to observations. We extend previous results showing that core collapse develops in an "outside-in" manner, with density and velocity approaching the Larson-Penston asymptotic solution. The time for the first core to collapse depends on Mach number as $t_{\text {coll }} \propto \mathcal{M}^{-1 / 2} \rho_{0}^{-1 / 2}$, for $\rho_{0}$ the mean pre-shock density, consistent with analytic estimates. Core building takes 10 times as long as core collapse, which lasts a few $\times 10^{5} \mathrm{yr}$, consistent with observed prestellar core lifetimes. Core shapes change from oblate to prolate as they evolve. To define cores, we use isosurfaces of the gravitational potential. We compare to cores defined using the potential computed from projected surface density, finding good agreement for core masses and sizes; this offers a new way to identify cores in observed maps. Cores with masses varying by three orders of magnitude $\left(\sim 0.05-50 M_{\odot}\right)$ are identified in our high- $\mathcal{M}$ simulations, with a much smaller mass range for models having low $\mathcal{M}$. We halt each simulation when the first core collapses; at that point, only the more massive cores in each model are gravitationally bound, with $E_{\text {th }}+E_{g}<0$. Stability analysis of post-shock layers predicts that the first core to collapse will have mass $M \propto v^{-1 / 2} \rho_{0}^{-1 / 2} T^{7 / 4}$, and that the minimum mass for cores formed at late times will have $M \propto v^{-1} \rho_{0}^{-1 / 2} T^{2}$, with $T$ being the temperature. From our simulations, the median mass lies between these two relations. At the time we halt the simulations, the $M$ versus $v$ relation is shallower for bound cores than unbound cores; with further evolution the small cores may evolve to become bound, steeping the $M$ versus $v$ relation.
\end{abstract}

Key words: ISM: structure - methods: numerical - stars: formation - turbulence

Online-only material: color figures

\section{INTRODUCTION}

Star formation begins with the creation of dense molecular cores, and understanding how cores grow and evolve is essential to identifying the origin of stellar properties (Shu et al. 1987; McKee \& Ostriker 2007; André et al. 2008). Through the 1990s, the prevailing theoretical picture was of slow core formation and evolution mediated by ambipolar diffusion, followed by core collapse initiated from a quasistatic, centrally concentrated state (e.g., Mouschovias 1987; Mouschovias \& Ciolek 1999). Current observations, however, indicate that magnetic field strengths are insufficient to provide the dominant support of molecular cores (Troland \& Crutcher 2008). In addition, over the past decade, a conception of star formation has emerged in which supersonic turbulence drives structure and evolution within giant molecular clouds (GMCs) on a wide range of scales (e.g., Ballesteros-Paredes et al. 2007; McKee \& Ostriker 2007). Because supersonic turbulence can compress gas to densities at which gravitational collapse can rapidly occur, it is likely to be important in the initiation of prestellar cores. Ultimately, models of core formation and evolution must take into account both moderate magnetic fields (with diffusion) and strong turbulence (Kudoh \& Basu 2008; Nakamura \& Li 2008). In order to gain insight into the physics involved, however, it is informative to focus on individual limiting cases and explore dependence on parameters. Here, following Gong \& Ostriker (2009) but generalizing to three dimensions, we consider core building and evolution in the turbulence-dominated, unmagnetized limit.
Observations of dense cores in GMCs have provided detailed information on individual core properties as well as statistics of core populations (see, e.g., the reviews of di Francesco et al. 2007; Ward-Thompson et al. 2007; Bergin \& Tafalla 2007; André et al. 2008). These properties, including internal structure and kinematics, durations of different evolutionary stages, and distribution of core masses, constrain core formation theories. In terms of structure, cores are observed to be centrally concentrated at all stages, with the specific profile fits differing depending on the stage of evolution. Cores can generally be fit with a uniform-density inner region surrounded by a power law $\propto r^{-2}$ (e.g., Shirley et al. 2000; Bacmann et al. 2000; Alves et al. 2001; Kandori et al. 2005; Kirk et al. 2005); this shape is consistent with expectations for both static Bonnor-Ebert (BE) pressure-supported isothermal equilibria (Bonnor 1956; Ebert 1955), and for collapsing isothermal spheres (Bodenheimer \& Sweigart 1968; Larson 1969; Penston 1969). The center-to-edge density contrast is frequently larger than the maximum possible for a stable BE sphere, however, and the inferred temperatures based on static BE fits are also often larger than observed temperatures. Although in principle some support could be provided by magnetic fields (e.g., Ciolek \& Mouschovias 1994), another possibility is that these "supercritical" cores are in fact collapsing rather than static (Dapp \& Basu 2009; Gong \& Ostriker 2009).

In terms of kinematics, dense, low-mass cores generally have subsonic internal velocity dispersions, whether for isolated cores or for cores found in clusters (e.g., Myers 1983; Goodman et al. 
1998; Caselli et al. 2002; Tafalla et al. 2004; Kirk et al. 2007; André et al. 2007; Lada et al. 2008). Some prestellar cores also show indications of subsonic inward motions throughout their interiors based on asymmetry of molecular lines that trace dense gas (e.g., Lee \& Myers 1999; Lee et al. 2001; Sohn et al. 2007). For cores containing protostars, signatures of supersonic inward motions on small scales $(\sim 0.01-0.1 \mathrm{pc})$ have been observed (e.g., Gregersen et al. 1997; Di Francesco et al. 2001); these are believed to be indicative of gravitationally induced infall. In very recent work, Pineda et al. (2010) have used $\mathrm{NH}_{3}$ observations to identify a sharp transition from supersonic to subsonic velocity dispersion from outer to inner regions in the core B5 in Perseus.

Several recent statistical studies have reached similar conclusions regarding the durations of successive stages of core evolution (e.g., Ward-Thompson et al. 2007; Enoch et al. 2008; Evans et al. 2009), with prestellar and protostellar (class 0) stages having comparable lifetimes. The typical duration for each of these stages is a few times the gravitational free-fall time

$$
t_{\mathrm{ff}}=\left(\frac{3 \pi}{32 G \bar{\rho}}\right)^{1 / 2}=4.3 \times 10^{5} \mathrm{yr}\left(\frac{\bar{n}_{\mathrm{H}}}{10^{4} \mathrm{~cm}^{-3}}\right)^{-1 / 2}
$$

at the mean core density $\bar{\rho}=1.4 m_{\mathrm{H}} \bar{n}_{\mathrm{H}}$, amounting to $\sim(1-5) \times 10^{5}$ yr for typical conditions. With prestellar lifetimes considerably below the ambipolar diffusion time for strong magnetic field $t_{\mathrm{AD}} \approx 10 t_{\mathrm{ff}}$ (e.g., Mouschovias \& Ciolek 1999), this suggests that observed cores are trans-critical or supercritical (see Ciolek \& Basu 2001) with respect to the magnetic field. ${ }^{1}$ This conclusion is also supported by magnetic field Zeeman observations (Troland \& Crutcher 2008), indicating that cores have mean mass-to-magnetic-flux ratios twice the critical value. Thus, magnetic field effects appear to be sub-dominant in terms of supporting cores against collapse, and ambipolar diffusion does not appear to control the dynamics of core formation and evolution. As magnetic fields are non-negligible, however, magnetohydrodynamic (MHD) stresses may still affect GMC and core dynamics.

Empirical measurements of core mass functions (CMFs; e.g., Motte et al. 1998, 2001; Testi \& Sargent 1998; Johnstone et al. 2000, 2001; Onishi et al. 2002; Beuther \& Schilke 2004; Reid \& Wilson 2005, 2006; Stanke et al. 2006; Enoch et al. 2006; Alves et al. 2007; Ikeda et al. 2007, 2009; Ikeda \& Kitamura 2009; Nutter \& Ward-Thompson 2007; Simpson et al. 2008; Könyves et al. 2010) show that CMFs have a remarkable similarity in shape to stellar initial mass functions (IMFs, see, e.g., Kroupa 2001; Chabrier 2005), with a shift toward lower mass by a factor of 3-4 (see, e.g., Alves et al. 2007; Rathborne et al. 2009). The characteristic/turnover mass of observed CMFs ranges from 0.1 to $3 M_{\odot}$, although there are uncertainties in this associated with lack of spatial resolution at the low-mass end.

Many theoretical efforts have contributed to interpreting the observed properties of cores. The classic work of Bonnor (1956) and Ebert (1955) provided the foundation of later studies, by determining the maximum mass of a static isothermal sphere that is dynamically stable. In terms of the boundary pressure $P_{\text {edge }}=\rho_{\text {edge }} c_{s}^{2}$ or mean internal density $\bar{\rho}=2.5 \rho_{\text {edge }}$, this

\footnotetext{
1 The critical mass-to-magnetic-flux defines the minimum that permits gravitational collapse in the field-freezing limit (e.g., Mestel \& Spitzer 1956; Mouschovias \& Spitzer 1976; Nakano \& Nakamura 1978).
}

maximum stable mass is

$$
\begin{aligned}
M_{\mathrm{BE}} & =1.2 \frac{c_{s}^{4}}{\left(G^{3} P_{\text {edge }}\right)^{1 / 2}}=1.9 \frac{c_{s}^{3}}{\left(G^{3} \bar{\rho}\right)^{1 / 2}} \\
& =2.3 M_{\odot}\left(\frac{\bar{n}_{\mathrm{H}}}{10^{4} \mathrm{~cm}^{-3}}\right)^{-1 / 2}\left(\frac{T}{10 \mathrm{~K}}\right)^{3 / 2} .
\end{aligned}
$$

Here, $c_{s}=(k T / \mu)^{1 / 2}$ is the internal sound speed in the core.

Over many years, numerical simulations have been used to investigate isothermal collapse of individual, pre-existing cores (Bodenheimer \& Sweigart 1968; Larson 1969; Penston 1969; Hunter 1977; Foster \& Chevalier 1993; Ogino et al. 1999; Hennebelle et al. 2003; Motoyama \& Yoshida 2003; Vorobyov \& Basu 2005; Gómez et al. 2007; Burkert \& Alves 2009). These simulations include initiation from static configurations that are unstable, and initiation from static, stable configurations that are subjected to imposed compression, either from enhanced external pressure or a converging velocity field, or a core-core collision. A common feature of the results is that the collapse generally starts from outside and propagates in as the central density increases. At the time of singularity formation, the density profile approaches the "Larson-Penston" asymptotic solution $\rho=8.86 c_{s}^{2} /\left(4 \pi G r^{2}\right)$ and the central velocity is comparable to the value $-3.28 c_{s}$ derived by Larson (1969) and Penston (1969). However, these previous studies have not considered core evolution within the larger context, in particular including the process of core formation. Since the formation process may affect later evolution, it is important to develop unified models.

At GMC scales, a number of groups have investigated the CMFs that result from numerical simulations of turbulent, selfgravitating systems (see, e.g., Klessen 2001; Gammie et al. 2003; Bonnell et al. 2003; Li et al. 2004; Tilley \& Pudritz 2004; Heitsch et al. 2008; Clark et al. 2008; Offner et al. 2008; Basu et al. 2009; Smith et al. 2009). These models have shown-for certain parts of parameter space-features that are in accord with observed CMFs: mass functions dominated by the low end with a peak and turnover near $1 M_{\odot}$, and a high-mass powerlaw slope (at least marginally) consistent with the Salpeter value. These simulations have not, however, had sufficient resolution to investigate the internal properties of individual cores that form. In addition, these studies have not quantified how the core masses depend on the large-scale properties of the turbulent medium (see below).

Taking the previous numerical simulations of individual cores one step further, Gong \& Ostriker (2009) initiated a study of dynamically induced core formation and evolution in supersonic converging flows, focusing on the spherical case. In these simulations, the density is initially uniform everywhere: no initial core structure is assumed. Instead, dense cores form inside a spherical shock that propagates outward within the converging flow. Over time, cores become increasingly stratified as their masses grow. Eventually, the core collapses to create a protostar following the same "outside-in" pattern as in models initiated from static conditions. Subsequently, the dense envelope falls into the center via an inside-out rarefaction wave (Shu 1977; Hunter 1977); this is followed by a stage of late accretion if the converging flow on large scales continues to be maintained. The unified formation and evolution model of Gong \& Ostriker (2009) explains many observed core properties, including BE-sphere-like density profiles, subsonic internal velocities within cores, and short core lifetimes with comparable prestellar and protostellar durations. Gong \& Ostriker (2009) also found 
that the inflow velocity of the converging flows affects core lifetimes, masses, sizes, and accretion histories. Realistic supersonic inflows in clouds are not spherical, however, while mass inflow rates are affected by geometry. Thus, the quantitative results for masses, lifetimes, etc., as a function of Mach number and ambient density may differ for more realistic geometry.

Numerical results on core formation have not reached consensus on how the characteristic mass in the $\mathrm{CMF}, M_{c}$, depends on the bulk properties of the cloud-its mean density $\rho_{0}=\langle\rho\rangle$, sound speed $c_{s}$, and turbulent velocity dispersion $v_{\text {turb }}$. Some have suggested that the Jeans mass of the cloud at its mean density $\left(M_{J}=c_{s}^{3} \pi^{3 / 2}\left(G^{3} \rho_{0}\right)^{-1 / 2}\right)$ determines $M_{c}$ in the CMF (e.g., Klessen 2001; Bonnell et al. 2006), while others have found values of $M_{c}$ well below $M_{J}$ (see, e.g., Gammie et al. 2003; Li et al. 2004). As noted by McKee \& Ostriker (2007), the difference between these conclusions is likely related to the Mach number of turbulence: the value found for $M_{c} / M_{J}$ is lower in simulations where the Mach number $\mathcal{M} \equiv v_{\text {turb }} / c_{s}$ is higher. Indeed, more recent simulations by Clark et al. (2008) provide some indication that increasing $\mathcal{M}$ lowers the value of $M_{c}$ in the CMF; they did not, however, conduct a full parameter study.

Supersonic turbulence makes the density in a GMC highly non-uniform, creating a log-normal probability distribution function (PDF) in which most of the volume is at densities below $\rho_{0}$ and most of the mass is at densities above $\rho_{0}$ (e.g., Vazquez-Semadeni 1994; Padoan et al. 1997; Ostriker et al. 1999). Given that the log-normal PDF allows for a range of Jeans masses (or BE masses; $M_{\mathrm{BE}} \propto M_{J}$ ), Padoan \& Nordlund (2002, 2004) proposed that the CMF is set by dividing the total available gas mass at each density into unstable cores. Padoan et al. (2007) propose that the peak mass in the CMF is given by $M_{c}=3 M_{\mathrm{BE}, 0} / M_{A}^{1.1}$ for $M_{A} \equiv v_{\text {turb }} / v_{A}$ the Alfvén Mach number in a cloud, and $M_{\mathrm{BE}, 0}$ the $\mathrm{BE}$ mass evaluated at the mean cloud density $n_{0}$. Here, $v_{A} \equiv B /(4 \pi \rho)^{1 / 2}$ is the Alfvén speed. For realistic mean GMC density $n_{0} \sim 100 \mathrm{~cm}^{-3}$ and $\mathcal{M}_{A} \sim 1-4$, from Equation (2) the Padoan et al. formula in fact yields $M_{c}>15 M_{\odot}$; only if one chooses a much higher reference density does this agree with observations. For the unmagnetized case, Padoan et al. (2007) propose that $M_{c}=4 M_{\mathrm{BE}, 0} / \mathcal{M}^{1.7}$. Hennebelle \& Chabrier (2008) point out that shock compression is underestimated in the magnetized case by Padoan et al. (2007), and advocate a formula similar to their unmagnetized one: $M_{c} \sim M_{\mathrm{BE}, 0} / \mathcal{M}^{3 / 2}$. Since $\mathcal{M} \gtrsim 10$ in massive GMCs, these formulae yield more realistic values $M_{c} \sim M_{\odot}$. Neither the Padoan et al. (2007) or the Hennebelle $\&$ Chabrier (2008) proposal has, however, been tested directly using self-gravitating numerical simulations.

In this contribution, we present results on core formation and evolution based on a large suite of three-dimensional (3D) numerical simulations. Each simulation models a localized region of a turbulent cloud in which there is an overall convergence in the velocity field. Under the assumption that there is a dominant convergence direction locally, we choose inflow along a single axis, so that convergence is planar. With the more realistic geometry afforded by the current simulations, we are able to check the results obtained by Gong \& Ostriker (2009) for core building and collapse in supersonic flows. We are also able to explore how the characteristic core mass is related to the velocity of the converging flows. Since the speed of converging flow is assumed to reflect the amplitude of the largest-scale (dominant) motions in a GMC, this relates the characteristic core mass to the turbulent Mach number in its parent GMC. Although a number of previous studies of core formation have been conducted, the present investigation is distinguished by our systematic study of Mach number dependence, together with our focus on internal structure and kinematics of the cores that form.

The outline of this paper is as follows. In Section 2, we provide a physical discussion of self-gravitating core formation in the post-shock dense layers, identifying the mass, size, and timescales expected to be important. In Section 3, we summarize the governing equations and methods used in our numerical simulations. Section 4 describes the development of core structure and evolution in our models, paying particular attention to the influence of Mach number $\mathcal{M}$ on the evolution, and comparing collapse of individual cores with Gong \& Ostriker (2009). Section 5 describes our method of core-finding, in which the largest closed contour of the gravitational potential determines the core size. We demonstrate that this method can be used for both three-dimensional and two-dimensional (2D) data with similar results, and can thus be applied to find cores in observed clouds. Section 6 describes the relations between core properties (core mass, core radius, and core collapse time) and the large-scale Mach number of the converging flow, relating to the expectations from gravitational instability discussed in Section 2. In Section 6, we also quantify core shapes, and explore the relationship between core structure and kinematics. Section 7 summarizes our new results and discusses our findings in the context of previous theories and observations.

\section{THE CHARACTERISTIC CORE MASS AND SIZE}

Prior to describing our numerical model prescription and results, it is useful to summarize the scales that are likely to be relevant for formation of self-gravitating cores in GMCs. We shall assume approximately isothermal conditions, consistent with observations (e.g., Blitz et al. 2007). The isothermal sound speed at a temperature $T$ is

$$
c_{s}=0.20 \mathrm{~km} \mathrm{~s}^{-1}\left(\frac{T}{10 \mathrm{~K}}\right)^{1 / 2} .
$$

If the density within clouds were uniform, the spatial scale relevant for gravitational instability would be the Jeans length

$$
L_{J} \equiv c_{s}\left(\frac{\pi}{G \rho_{0}}\right)^{1 / 2}=2.76 \mathrm{pc}\left(\frac{n_{\mathrm{H}, 0}}{10^{2} \mathrm{~cm}^{-3}}\right)^{-1 / 2}\left(\frac{T}{10 \mathrm{~K}}\right)^{1 / 2}
$$

evaluated at the mean density $\rho_{0}$. The corresponding Jeans mass is

$$
\begin{aligned}
M_{J} \equiv \rho_{0} L_{J}^{3} & =c_{s}^{3}\left(\frac{\pi^{3}}{G^{3} \rho_{0}}\right)^{1 / 2} \\
& =72 M_{\odot}\left(\frac{n_{\mathrm{H}, 0}}{10^{2} \mathrm{~cm}^{-3}}\right)^{-1 / 2}\left(\frac{T}{10 \mathrm{~K}}\right)^{3 / 2}
\end{aligned}
$$

Note that $\rho_{0}\left(L_{J} / 2\right)^{3}$ or $\rho_{0} 4 \pi\left(L_{J} / 2\right)^{3} / 3$ is sometimes used for the Jeans mass. The BE mass (Equation (2)) for $P_{\text {edge }}=P_{0} \equiv$ $\rho_{0} c_{s}^{2}$ is $M_{\mathrm{BE}}=0.22 M_{J}\left(\rho_{0}\right)$. The Jeans time at the mean cloud density is

$$
\begin{aligned}
t_{J} \equiv \frac{L_{J}}{c_{s}} & =\left(\frac{\pi}{G \rho_{0}}\right)^{1 / 2}=3.27 t_{\mathrm{ff}}\left(\rho_{0}\right) \\
& =1.4 \times 10^{7} \mathrm{yr}\left(\frac{n_{\mathrm{H}, 0}}{10^{2} \mathrm{~cm}^{-3}}\right)^{-1 / 2} .
\end{aligned}
$$

We shall use the Jeans length, mass, and time at the unperturbed 
density as our code units of length, mass, and time: $L_{0}=L_{J}$, $M_{0}=M_{J}$, and $t_{0}=t_{J}$.

Of course, GMCs are highly inhomogeneous, with core formation taking place in the overdense regions that have the shortest gravitational times. If the overdense regions within GMCs are produced by shocks in the turbulent, supersonic flow, their density, and therefore the mass scale and length scale for growth of self-gravitating structures, will be related to the shock strength. Strongly magnetized shocks have less compression than weakly magnetized shocks (while both will be present in a turbulent flow), so we concentrate on the latter case.

If gravitationally unstable cores develop only in gas that has been strongly compressed by shocks, the actual bounding pressure will be much larger than $P_{0}=\rho_{0} c_{s}^{2}$. In particular, an isothermal shock with Mach number $\mathcal{M}$ will produce a postshock region with pressure $P_{\text {post-shock }}=\rho_{0} v^{2}=\mathcal{M}^{2} \rho_{0} c_{s}^{2} \gg$ $P_{0}$. Thus, if cores preferentially form in stagnation regions between shocks of Mach number $\mathcal{M}$, then one can define an effective BE mass for these core-forming regions within the turbulent flow by setting $P_{\text {edge }}=P_{\text {post-shock }}$ in Equation (2):

$$
\begin{aligned}
M_{\mathrm{BE}, \text { post-shock }} \equiv & 1.2 \frac{c_{s}^{3}}{\left(G^{3} \rho_{0}\right)^{1 / 2}} \frac{1}{\mathcal{M}}=2.8 M_{\odot}\left(\frac{v}{1 \mathrm{~km} \mathrm{~s}^{-1}}\right)^{-1} \\
& \times\left(\frac{n_{\mathrm{H}, 0}}{10^{2} \mathrm{~cm}^{-3}}\right)^{-1 / 2}\left(\frac{T}{10 \mathrm{~K}}\right)^{2} .
\end{aligned}
$$

The above simple argument suggests $M \propto v^{-1} \rho_{0}^{-1 / 2} T^{2}$ for the minimum mass of a star that forms via collapse of a core in a turbulent cloud with velocity dispersion $v$, mean density $\rho_{0}$, and temperature $T$.

Equation (7) provides a mass scale for fragmentation within post-shock regions, but in fact instabilities take some time to develop. Thus, it is useful to consider the evolution of a simple system consisting of a planar shocked layer formed by a converging flow (see, e.g., Elmegreen \& Elmegreen 1978; Lubow \& Pringle 1993; Vishniac 1994; Whitworth et al. 1994; Iwasaki \& Tsuribe 2008).

For inflow Mach number $\mathcal{M}$, the surface density of the postshock layer at time $t$ is

$$
\Sigma(t)=\rho_{0}\left(v_{z,+}-v_{z,-}\right) t=2 \rho_{0} \mathcal{M} c_{s} t,
$$

where $v_{z,+}$ and $v_{z,-}$ are the upward and downward converging velocities. If the sheet is not vertically self-gravitating, its halfthickness is $H=\Sigma(t) / 2 \rho_{p}$ where $\rho_{p} \approx \rho_{0} \mathcal{M}^{2}$ is the post-shock density. The non-self-gravitating half-thickness is thus

$$
H_{\mathrm{nsg}} \approx \frac{2 \rho_{0} \mathcal{M} c_{s} t}{2 \rho_{0} \mathcal{M}^{2}}=\frac{c_{s} t}{\mathcal{M}} .
$$

As the surface density of the sheet increases, self-gravity will become increasingly important in confining the gas. In the limit of hydrostatic equilibrium, the height approaches

$$
H_{\mathrm{sg}}=\frac{c_{s}^{2}}{\pi G \Sigma}=\frac{c_{s}}{2 \pi G \rho_{0} \mathcal{M} t} .
$$

Note that the transition from non-self-gravitating $\left(H_{\mathrm{nsg}} \propto t\right)$ to self-gravitating $\left(H_{\mathrm{sg}} \propto t^{-1}\right)$ occurs at a time near

$$
t_{\mathrm{sg}} \equiv \frac{1}{\left(2 \pi G \rho_{0}\right)^{1 / 2}}=0.22 t_{J}
$$

defined by the condition $H_{\mathrm{sg}}=H_{\mathrm{nsg}}$.
The dispersion relation for in-plane modes in a slab, allowing for non-zero $H$ (e.g., Kim et al. 2002), is

$$
\omega^{2} \approx c_{s}^{2} k^{2}-\frac{2 \pi G \Sigma k}{1+k H}
$$

For the critical mode $\omega^{2}=0$, so that

$$
k_{\text {crit }} H\left(1+k_{\text {crit }} H\right)=2 \pi H \frac{G \Sigma}{c_{s}^{2}}=2 \pi \frac{H}{L_{\mathrm{J}, 2 \mathrm{D}}},
$$

where

$$
L_{\mathrm{J}, 2 \mathrm{D}} \equiv \frac{c_{s}^{2}}{G \Sigma}
$$

is the Jeans length for an infinitesimally thin layer. The solution to Equation (13) is

$$
\begin{aligned}
k_{\mathrm{crit}} & =\frac{2 \pi}{L_{\mathrm{J}, 2 \mathrm{D}}} \frac{2}{1+\left(1+8 \pi \frac{H}{L_{\mathrm{J}, 2 \mathrm{D}}}\right)^{1 / 2}} \\
& =\frac{4 \pi G \rho_{0} t \mathcal{M}}{c_{s}} \frac{2}{1+\left(1+8 \pi \frac{H}{L_{\mathrm{J}, 2 \mathrm{D}}}\right)^{1 / 2}},
\end{aligned}
$$

so that

$$
\begin{aligned}
\lambda_{\text {crit }} & =L_{\mathrm{J}, 2 \mathrm{D}} \frac{1+\left(1+8 \pi \frac{H}{L_{\mathrm{J}, 2 \mathrm{D}}}\right)^{1 / 2}}{2} \\
& =\frac{c_{s}}{2 G \rho_{0} t \mathcal{M}} \frac{1+\left(1+8 \pi \frac{H}{L_{\mathrm{J}, 2 \mathrm{D}}}\right)^{1 / 2}}{2} .
\end{aligned}
$$

The corresponding critical mass $\left(\lambda_{\text {crit }} / 2\right)^{2} \Sigma$ is

$$
M_{\text {crit }} \equiv \frac{c_{s}^{3}}{32 G^{2} \rho_{0} \mathcal{M}} \frac{\left[1+\left(1+8 \pi \frac{H}{L_{\mathrm{J}, 2 \mathrm{D}}}\right)^{1 / 2}\right]^{2}}{t} .
$$

Note that $H / L_{\mathrm{J}, 2 \mathrm{D}}$ initially increases in time, during the nonself-gravitating stage $\left(H_{\mathrm{nsg}} / L_{\mathrm{J}, 2 \mathrm{D}}=2 G \rho_{0} t^{2}\right)$, and then approaches a constant $\left(H_{\mathrm{sg}} / L_{\mathrm{J}, 2 \mathrm{D}}=1 / \pi\right)$. At any time, all wavelengths $\lambda>\lambda_{\text {crit }}$ have $\omega^{2}<0$, so that overdense regions of the corresponding sizes and masses $M>M_{\text {crit }}$ grow relative to their surroundings.

During the non-self-gravitating stage, the critical mass has a minimum value at time

$$
t_{\text {crit,nsg, } \min }=\left(\frac{3}{16 \pi G \rho_{0}}\right)^{1 / 2}=0.14 t_{J}=0.61 t_{\mathrm{sg}}
$$

given by

$$
\begin{gathered}
M_{\text {crit,nsg, } \min }=\frac{3 \sqrt{3 \pi}}{8} \frac{c_{s}^{3}}{\left(G^{3} \rho_{0}\right)^{1 / 2}} \frac{1}{\mathcal{M}} \\
=3.0 M_{\odot}\left(\frac{v}{1 \mathrm{~km} \mathrm{~s}^{-1}}\right)^{-1}\left(\frac{n_{\mathrm{H}, 0}}{10^{2} \mathrm{~cm}^{3}}\right)^{-1 / 2}\left(\frac{T}{10 \mathrm{~K}}\right)^{2} .
\end{gathered}
$$

The numerical coefficient in Equation (19) is 1.15; note that this is almost the same as in Equation (7).

At late time, the critical mass from Equation (17) with $H_{\mathrm{sg}} / L_{\mathrm{J}, 2 \mathrm{D}}=1 / \pi$ becomes

$$
M_{\text {crit,sg }}=\frac{c_{s}^{3}}{2 G^{2} \rho_{0} \mathcal{M} t}=\frac{c_{s}^{4}}{G^{2} \Sigma} .
$$


Expressing $M_{\text {crit,sg }}$ in terms of the virial parameter $\alpha_{\text {vir }}=$ $5 \sigma_{v}^{2} R / G M_{\mathrm{GMC}}$ of the GMC, and using $\sigma_{v}=\mathcal{M} c_{s}$ and $M_{\mathrm{GMC}}=$ $\pi R^{2} \Sigma_{\mathrm{GMC}}=4 \pi R^{3} \rho_{0} / 3$, we have

$$
M_{\text {crit }, \mathrm{sg}}=\left(\frac{3 \pi \alpha_{\mathrm{vir}}}{20}\right)^{1 / 2} \frac{c_{s}^{3}}{\left(G^{3} \rho_{0}\right)^{1 / 2}} \frac{1}{\mathcal{M}} \frac{\Sigma_{\mathrm{GMC}}}{\Sigma} .
$$

Here, $\sigma_{v}$ is the large-scale one-dimensional (1D) velocity dispersion in GMCs, which will be responsible for the largestscale, strongest shocks. Taking $\alpha_{\text {vir }}=2$, the coefficient in Equation (22) is 0.97 , so this is very similar to Equations (7) and (19) if $\Sigma \sim \Sigma_{\mathrm{GMC}}$. In dimensional units, the critical mass (for $\Sigma=\Sigma_{\mathrm{GMC}}$ ) is

$$
M_{\text {crit,sg }}=2.5 M_{\odot}\left(\frac{v}{1 \mathrm{~km} \mathrm{~s}^{-1}}\right)^{-1}\left(\frac{n_{\mathrm{H}, 0}}{10^{2} \mathrm{~cm}^{-3}}\right)^{-1 / 2}\left(\frac{T}{10 \mathrm{~K}}\right)^{2}
$$

As noted above, Equations (7), (19)-(20), and (22)-(23) all have a similar form. An important task for numerical simulations is therefore to test the hypothesis that the characteristic mass scale of collapsing cores formed in turbulent, self-gravitating GMCs follows this scaling, i.e.,

$$
\begin{aligned}
M_{c} & =\psi \frac{c_{s}^{4}}{\left(G^{3} \sigma_{v}^{2} \rho_{0}\right)^{1 / 2}} \\
& =\psi \times 2.6 M_{\odot}\left(\frac{\sigma_{v}}{1 \mathrm{~km} \mathrm{~s}^{-1}}\right)^{-1}\left(\frac{n_{\mathrm{H}, 0}}{10^{2} \mathrm{~cm}^{-3}}\right)^{-1 / 2}\left(\frac{T}{10 \mathrm{~K}}\right)^{2},
\end{aligned}
$$

where $\psi$ is a dimensionless coefficient.

The critical mass given above is the smallest mass that can collapse, given infinite time. Since the growth rate depends on scale (and is formally zero for critical perturbations), at any finite time only cores that have grown sufficiently rapidly will be nonlinear enough to collapse. It is therefore useful to consider how much growth has occurred at a given time. Consider a perturbation of wavenumber $k$ that instantaneously has $d^{2} \delta \Sigma / d t^{2}=-\omega^{2} \delta \Sigma$ so that $\delta \Sigma=\delta \Sigma_{\text {init }} e^{\Gamma}$ where $\Gamma=$ $\ln \left(\delta \Sigma / \delta \Sigma_{\text {init }}\right)=\int\left(-\omega^{2}\right)^{1 / 2} d t$. Using Equation (12),

$$
\Gamma=\int_{t_{\min }}^{t}\left(-\omega^{2}\right)^{1 / 2} d t=\int_{t_{\min }}^{t}\left(\frac{2 \pi G \Sigma k}{1+k H}-c_{s}^{2} k^{2}\right)^{1 / 2} d t
$$

where $t_{\min }$ is the instant when $\Sigma$ is large enough that perturbations of wavenumber $k$ start to grow $\left(-\omega^{2} \geqslant 0\right)$. With $\Sigma=2 \rho_{0} c_{s} \mathcal{M}$, $t_{\text {min }}=c_{s} k(1+k H) /\left(4 \pi G \rho_{0} \mathcal{M}\right)$. If we assume $k H \ll 1$ (see below), then

$$
\Gamma=\frac{2 \sqrt{2}}{3} \kappa^{1 / 2}(\tau-\kappa / 2)^{3 / 2},
$$

where $\kappa=k c_{s} / \sqrt{2 \pi G \rho_{0} \mathcal{M}}$ and $\tau=t \sqrt{2 \pi G \rho_{0} \mathcal{M}}$.

At a given time $t$ (or $\tau$ ) during the evolution, the mode $k_{m}$ (or $\kappa_{m}$ ) that has grown the most has $\partial \ln \Gamma / \partial k=0$, which gives

$$
\kappa_{m}=\frac{\tau}{2}
$$

and $\Gamma_{\max }=\Gamma\left(k_{m}\right)=\sqrt{3} \kappa_{m}^{2}=\sqrt{3} \pi G \rho_{0} \mathcal{M} t^{2} / 2$. The mass of this most-amplified mode is

$$
M_{m} \equiv\left(\frac{\lambda_{m}}{2}\right)^{2} \Sigma=\left(\frac{2 \sqrt{3} \pi}{\Gamma_{\max }}\right)^{1 / 2} \frac{c_{s}^{3}}{\left(G^{3} \rho_{0}\right)^{1 / 2}} \frac{1}{\mathcal{M}^{1 / 2}},
$$

where the time is

$$
t=\left(\frac{2 \Gamma_{\max }}{\sqrt{3} \pi}\right)^{1 / 2}\left(\frac{1}{G \rho_{0}}\right)^{1 / 2} \frac{1}{\mathcal{M}^{1 / 2}},
$$

and $k_{m}=\left(\Gamma_{\max } / \sqrt{3}\right)^{1 / 2}\left(2 \pi G \rho_{0} \mathcal{M}\right)^{1 / 2} / c_{s}$, so that

$$
\lambda_{m}=\left(\frac{2 \sqrt{3} \pi}{\Gamma_{\max }}\right)^{1 / 2} \frac{c_{s}}{\left(G \rho_{0}\right)^{1 / 2}} \frac{1}{\mathcal{M}^{1 / 2}} .
$$

With $\Gamma_{\max }=1$, the numerical coefficient for $M_{m}$ in Equation (28) is 3.30, and Equation (29) gives $t=$ $0.34 t_{J} \mathcal{M}^{-1 / 2}$, corresponding to $\tau=1.5$. Note that for low Mach number, this time exceeds $t_{\text {sg }}$ (see Equation (11)), whereas for high Mach number it does not. Also, note that with $H<$ $c_{s} t_{\mathrm{sg}} / \mathcal{M} \equiv H_{\max }$ (see Equations (9)-(11)), $k_{m} H<k_{m} H_{\max }=$ $\Gamma_{\max }^{1 / 2}(\sqrt{3} \mathcal{M})^{-1 / 2}$. Taking $\Gamma_{\max }=1, k_{m} H<0.8$ for $\mathcal{M}>1$, with $k_{m} H \ll 1$ for $\mathcal{M} \gg 1$. This verifies self-consistency of the assumption made in obtaining Equation (26).

Written in terms of $v, \rho_{0}$, and $T$, the most-amplified mass is

$$
\begin{aligned}
M_{m}= & 19.1 M_{\odot}\left(\frac{v}{1 \mathrm{~km} \mathrm{~s}^{-1}}\right)^{-1 / 2}\left(\frac{n_{\mathrm{H}, 0}}{10^{2} \mathrm{~cm}^{-3}}\right)^{-1 / 2} \\
& \times\left(\frac{T}{10 \mathrm{~K}}\right)^{7 / 4}\left(\Gamma_{\max }\right)^{-1 / 2}
\end{aligned}
$$

Comparing Equation (31) with Equation (23), we see that a different dependence on velocity (or Mach number) is expected for the first core to collapse (Equation (31)), compared with the dependence for a typical core to eventually form (Equation (23)). Similar results to Equation (28) have previously been discussed by other authors. Whitworth et al. (1994) point out that the fastest-growing scale $\sim L_{\mathrm{J}, 2 \mathrm{D}} \sim$ $c_{s} /\left(G \rho_{0} \mathcal{M} t\right)$ will become nonlinear if the time exceeds the growth time $\sim L_{\mathrm{J}, 2 \mathrm{D}} / c_{s} \sim\left(G \rho_{0} \mathcal{M} t\right)^{-1}$, which occurs for $t \sim\left(G \rho_{0} \mathcal{M}\right)^{-1 / 2}$ (cf. our Equation (29)). This corresponds to a length scale $L_{\text {fragment }} \sim c_{s}\left(G \rho_{0} \mathcal{M}\right)^{-1 / 2}$ (cf. our Equation (30)), and a mass scale $M_{\text {fragment }} \sim c_{s}^{3}\left(G^{3} \rho_{0} \mathcal{M}\right)^{-1 / 2}$ (cf. our Equation (28)). By direct integration of the perturbation equation of the converging-flow system, Iwasaki \& Tsuribe (2008) find that the fastest-growing mode becomes nonlinear at time $0.96 \delta_{0}^{-0.1}\left(G \rho_{0} \mathcal{M}\right)^{-1 / 2}$, for $\delta_{0}$ the initial amplitude (cf. our Equation (29), which has a coefficient 0.6 if $\Gamma_{\max }=1$ ).

Finally, we note that the characteristic mass scale at late times given in Equation (24) can be connected to observed core mass scales using the empirical relationships among turbulence level, size, and mass for GMCs. In terms of the viral parameter $\alpha_{\mathrm{vir}} \equiv 5 \sigma_{v}^{2} R /\left(G M_{\mathrm{GMC}}\right)$ and the GMC surface density $\Sigma_{\mathrm{GMC}} \equiv 4 \rho_{0} R / 3$, Equation (24) can be re-expressed as

$$
\begin{aligned}
M_{c} & =1.5 \psi \frac{c_{s}^{4}}{\alpha_{\mathrm{vir}}^{1 / 2} G^{2} \Sigma_{\mathrm{GMC}}} \\
& =\psi \times 1 M_{\odot}\left(\frac{T}{10 \mathrm{~K}}\right)^{2}\left(\frac{\Sigma_{\mathrm{GMC}}}{100 M_{\odot} \mathrm{pc}^{-2}}\right)^{-1} \alpha_{\mathrm{vir}}^{-1 / 2} .
\end{aligned}
$$

With $\alpha_{\text {vir }} \sim 1-2$ and $\Sigma_{\mathrm{GMC}} \sim 100 M_{\odot} \mathrm{pc}^{-2}$ for observed clouds (Solomon et al. 1987; McKee \& Ostriker 2007; Heyer et al. 2009), the mass scale is intriguingly similar to the characteristic (peak) mass of CMFs within nearby molecular clouds. This relation potentially also offers a prediction for the peak of the CMF (and ultimately the IMF) when stars form under conditions 
different from those in most Milky Way GMCs. In particular, high temperature (up to $\sim 70 \mathrm{~K}$ ) may hold in starburst regions where the radiation field is strong and turbulent dissipation rates are high; since the temperature dependence of Equation (32) is steeper than the dependence on surface density, this could imply higher masses under those conditions.

\section{METHODS FOR NUMERICAL SIMULATIONS}

The numerical simulations we present here are conducted with the Athena code (Gardiner \& Stone 2005, 2008; Stone et al. 2008; Stone \& Gardiner 2009), using the HLLC solver (Toro 1999) and second-order reconstruction (Stone et al. 2008). To calculate the self-gravity of our slab domains, which are periodic in-plane and open in the $z$-direction, the fast Fourier transformation (FFT) method developed by Koyama \& Ostriker (2009) is used. We solve the three-dimensional equations of hydrodynamics,

$$
\begin{gathered}
\frac{\partial \rho}{\partial t}+\nabla \cdot(\rho \boldsymbol{v})=0 \\
\frac{\partial \boldsymbol{v}}{\partial t}+\boldsymbol{v} \cdot \nabla \boldsymbol{v}=-\frac{\nabla P}{\rho}-\nabla \Phi
\end{gathered}
$$

and the Poisson equation,

$$
\nabla^{2} \Phi=4 \pi G \rho
$$

where $\Phi$ is the gravitational potential. The isothermal assumption $P=c_{s}^{2} \rho$ is adopted. Pavlovski et al. (2006) found the isothermal approximation is adequate for simulations of the interstellar medium even with strong turbulence, which implies strong shocks in GMCs.

The code unit of density $\rho_{0}$ is a fiducial density representing the volume-averaged ambient density in a cloud on large scales; this characterizes the mean density of converging flows. For the code unit of velocity, we adopt the isothermal sound speed $c_{s}$ (see Equation (3)). For the unit of length, we adopt $L_{0}=L_{J}$, the Jeans length at the fiducial density (see Equation (4)). The mass and time units for the simulation are then $M_{0}=M_{J}$ (see Equation (5)) and $t_{0}=t_{J}$ (see Equation (6)).

In making comparison to observations, the total surface density integrated through the domain

$$
\Sigma=\int \rho(x, y, z) d z=\Sigma_{0} \int \frac{\rho}{\rho_{0}} \frac{d z}{L_{J}}
$$

is useful, for $\Sigma_{0} \equiv \rho_{0} L_{J}=9.49 M_{\odot} \mathrm{pc}^{-2}(T / 10 \mathrm{~K})^{1 / 2}\left(\mathrm{n}_{\mathrm{H}, 0} /\right.$ $\left.10^{2} \mathrm{~cm}^{-3}\right)^{1 / 2}$. In terms of the column density of hydrogen,

$$
N_{\mathrm{H}}=\frac{\Sigma}{1.4 m_{p}}=N_{0} \int \frac{n_{H}}{n_{\mathrm{H}, 0}} \frac{d z}{L_{J}}
$$

for $N_{0} \equiv n_{0} L_{J}=8.51 \times 10^{20} \mathrm{~cm}^{-2}(T / 10 \mathrm{~K})^{1 / 2}\left(\mathrm{n}_{\mathrm{H}, 0} /\right.$ $\left.10^{2} \mathrm{~cm}^{-3}\right)^{1 / 2}$. The mean line-of-sight velocity is calculated by

$$
\left\langle v_{\mathrm{los}}\right\rangle=\frac{\int \rho v_{\mathrm{los}} d s}{\int \rho d s},
$$

and the corresponding dispersion of $\left\langle v_{\text {los }}\right\rangle$ is defined as

$$
\sigma_{\mathrm{los}}^{2}=\frac{\int \rho\left(v_{\mathrm{los}}-\left\langle v_{\mathrm{los}}\right\rangle\right)^{2} d s}{\int \rho d s},
$$

where $d s=\sec \theta d z$ and $\theta$ is the tilt angle of the observer with respect to the $z$-axis.

Our model prescription consists of a converging flow augmented with turbulent velocity perturbations. In our parameter survey, the Mach number $\mathcal{M}$ of the inflow velocity ranges from 1.1 to 9 . Thus, two flows converge toward the central plane $z=0$ from the upper $z$-boundary (with mean velocity $-\mathcal{M} c_{s}$ ) and the lower $z$-boundary (with mean velocity $\mathcal{M} c_{s}$ ). The initial density is uniform and set to $\rho_{0}$, and the density at the inflowing $z$-boundaries is also set to $\rho_{0}$ throughout the simulation. The boundaries in the $x$ - and $y$-directions are periodic.

For both the whole domain initially and the inflowing gas subsequently, we apply perturbations following a Gaussian random distribution, with a Fourier power spectrum of the form

$$
\left\langle\left|\delta \boldsymbol{v}_{k}\right|^{2}\right\rangle \propto k^{-2}
$$

for $|k L / 2 \pi|<N / 2$, where $N$ is the resolution and $L$ is the size of the simulation box in $x$ and $y$. The power spectrum is appropriate for supersonic turbulence as observed in GMCs (McKee \& Ostriker 2007). The perturbation velocity fields are pre-generated with resolution $256^{3}$ in a box of size $L^{3}$. The perturbation fields are advected inward from the $z$-boundaries at inflow speed $\mathcal{M} c_{s}$ : at time intervals $\Delta t=\Delta z /\left(\mathcal{M} c_{s}\right)$, slices of the pre-generated perturbation fields for $v_{x}, v_{y}$, and $v_{z}$ are read in to update values in the ghost zones at the $z$-boundaries.

In addition to exploring dependence on the mean inflow Mach number $\mathcal{M}$, we also test dependence on the amplitude of turbulent perturbations on top of this converging flow. From the scaling law (see, e.g., Larson 1981; Heyer \& Brunt 2004) of self-gravitating molecular clouds, $\delta v(l) \propto l^{1 / 2}$, we can write the velocity dispersion at scale $l$ in terms of cloud-scale one-dimensional velocity dispersion $\sigma_{v}$ and cloud radius $R$ as $\delta v_{1 \mathrm{D}}(l)=\sigma_{v}(l / 2 R)^{1 / 2}$. The velocity dispersion at the scale of the simulation box $L$ is

$$
\frac{\delta v_{1 \mathrm{D}}(L)}{c_{s}}=\frac{\sigma_{v}}{c_{s}}\left(\frac{L}{2 R}\right)^{1 / 2}=\frac{\sigma_{v}}{c_{s}}\left(\frac{L}{L_{J}}\right)^{1 / 2}\left(\frac{2 R}{L_{J}}\right)^{-1 / 2} .
$$

In terms of the viral parameter $\alpha_{\text {vir }} \equiv 5 \sigma_{v}^{2} R /(G M)$, where $M=4 \pi R^{3} \rho_{0} / 3$ is the cloud mass, the ratio between $\sigma_{v}$ and $c_{s}$ is

$$
\frac{\sigma_{v}}{c_{s}}=2 \pi\left(\frac{\alpha_{\mathrm{vir}}}{15}\right)^{1 / 2} \frac{R}{L_{J}} .
$$

Solving Equation (42) for $2 R / L_{J}$ and substituting into Equation (41), we have the amplitude of perturbation for the simulation box:

$$
\frac{\delta v_{1 \mathrm{D}}(L)}{c_{s}}=\left(\frac{\alpha_{\mathrm{vir}} \pi^{2}}{15}\right)^{1 / 4}\left(\frac{\sigma_{v}}{c_{s}}\right)^{1 / 2}\left(\frac{L}{L_{J}}\right)^{1 / 2} .
$$

Thus, if the size of the simulation box is $L=L_{J}$ and $\alpha_{\text {vir }}=$ $1-2$, the perturbation amplitude would be

$$
\frac{\delta v_{1 \mathrm{D}}\left(L_{J}\right)}{c_{s}} \approx\left(\frac{\sigma_{v}}{c_{s}}\right)^{1 / 2}
$$

If we take the Mach number of the inflow, $\mathcal{M}$, as comparable to the value $\sigma_{v} / c_{s}$ of the whole cloud, then Equation (44) implies that higher converging velocities would be associated with higher amplitudes for the perturbation fields, for a given simulation box size $L_{J}$. To test the influence of the perturbation amplitude, we conduct two sets of simulations with $10 \%$ and 
$100 \%$ of the value $\delta v_{1 \mathrm{D}}\left(L_{J}\right)=(\mathcal{M} / 3)^{1 / 2} c_{S}$. Hereafter, we denote these cases as low-amplitude and high-amplitude initial perturbations, respectively.

For each Mach number $\mathcal{M}$ at each amplitude, we run 20 simulations with different random realizations of the same perturbation power spectrum, in order to collect sufficient statistical information on the core properties that result. The whole set of simulations therefore consists of 180 separate runs. The resolution for low-amplitude perturbation simulations is $N_{x} \times N_{y} \times N_{z}=256 \times 256 \times 96$, with domain size $L_{x} \times L_{y} \times L_{z} / L_{J}^{3}=1 \times 1 \times 0.375$; for high amplitude the resolution is $N_{x} \times N_{y} \times N_{z}=256 \times 256 \times 160$, with domain size $L_{x} \times L_{y} \times L_{z} / L_{J}^{3}=1 \times 1 \times 0.625$. The domain in the $z$-direction is smaller than in the $x$ - and $y$-directions since the reversed shock generated by the inflow only propagates a relatively short distance and the post-shock dense layer is thin, i.e., the basic geometry remains planar. The domain in the $z$-direction is large enough so that the post-shock layer does not evolve to reach the $z$-boundaries.

We note that our assumption of perturbed velocities but uniform densities in the inflowing gas is not fully realistic, since the flow entering a strong shock within a GMC will in general have internal density structure. ${ }^{2}$ In fact, the velocity perturbations we introduce do lead to moderate (order-unity) density fluctuations, as we have found by conducting comparison simulations with self-gravity turned off. These density fluctuations are what seed the growth of self-gravitating structures. The main emphasis of the current work is to investigate how the development of self-gravitating structures depends on the inflow Mach number, which sets the mean density (and hence the gravitational timescale) in the post-shock layer; previous studies have not tested the Mach number dependence of gravitational fragmentation. By varying the velocity perturbation amplitudes of the inflow, we have begun to explore the effect of pre-existing density structure on self-gravitating core development in shocked regions. This exploration can be extended and made more realistic (in terms of upstream structure) by investigating internal evolution of shocked layers within larger fully turbulent clouds having a range of mean Mach number; we are currently pursuing a numerical study along these lines. The models presented here may be thought of as investigating self-gravitating structure growth within the first strong shocks to develop inside a cloud.

\section{DEVELOPMENT OF STRUCTURE AND CORE EVOLUTION}

As discussed in Section 1, Gong \& Ostriker (2009) proposed a unified model for core formation and evolution in supersonic turbulent environments. Based on spherical-symmetry numerical simulations, four stages were identified: core building, core collapse, envelope infall, and late accretion. The duration of each stage, and the structure and kinematics of cores at varying stages were also analyzed. While the comparison of those results to observations is very encouraging, the assumption of spherical symmetry is clearly unrealistic. One of the key goals of this work is to check if core building and collapse still develop in a similar manner when the spherical-symmetry assumption is relaxed. Because the time step becomes very short in late stages, we halt the simulations; thus the current models do not address envelope infall and late accretion stages.

\footnotetext{
2 Other recent simulations of post-shock structure formation in converging flows have similarly assumed uniform density for the inflow (see, e.g., Heitsch et al. 2008; Banerjee et al. 2009, and references therein).
}

Figure 1 shows evolution of the surface density (Equation (36)) for models with $\mathcal{M}=1.1$ (left column), $\mathcal{M}=5$ (middle column), and $\mathcal{M}=8$ (right column), all with same realization for the perturbation velocities. The top panel of each column shows the surface density very early on; the patterns are identical but the amplitudes are different. The bottom panel shows the surface density when the most evolved core collapses for each case. Hereafter, we shall use $t_{\text {coll }}$ to denote the total time to reach collapse of the most evolved core, in terms of the code unit $t_{0}$ (Equation (6)). The four images from top to bottom in the same column show the surface density at four instants: $t=0.001 t_{0}, 1 / 3 t_{\text {coll }}, 2 / 3 t_{\text {coll }}$, and $t_{\text {coll }}$. Note that $t_{\text {coll }}=0.636 t_{0}, 0.280 t_{0}$, and $0.232 t_{0}$ for the $\mathcal{M}=1.1,5$, and 8 , respectively. These three simulations have low initial perturbation amplitude (cf. Equation (44)).

From Figure 1, two features are immediately apparent. First, the input perturbation field patterns determine the later structural evolution and there is a "family resemblance" for the models at different Mach number. This is because the post-shock dense layer retains a memory of the perturbation velocity fields in the direction parallel to the plane of the layer since $v_{x}$ and $v_{y}$ are unchanged across the shock interface. Comparing the first plot to the last plot of each column, cores form in regions where the density perturbation amplitudes are initially higher than the surroundings as a result of convergence in the $x-y$ plane. These overdense regions develop into long, thin filaments, within which cores grow and then collapse.

Second, the specific properties of cores, such as the total number and individual volumes (as well as their masses), are determined by $\mathcal{M}$. The dense cores for $\mathcal{M}=1.1$ are smoother than the cores for $\mathcal{M}=8$, and they cover larger areas. During the middle and late stages of evolution, more small-scale filamentary structures are evident in the higher Mach number cases. At a given scale, the input $v_{x}$ and $v_{y}$ perturbations are higher for larger $\mathcal{M}$, with the resulting compressions making more prominent "burrs" around cores. The "burrs" are also less smoothed for the high Mach number cases, because the shorter free-fall time at the higher post-shock density means that the core collapses sooner. Thus, as the velocity of the converging flow and additional perturbations increases, the result is smaller, denser, more irregular, and more "hairy" cores.

Figure 2 shows evolution of surface density and the mean in-plane velocities $\left\langle v_{x}\right\rangle$ and $\left\langle v_{y}\right\rangle$ for the $\mathcal{M}=5$ model shown in Figure 1. The mean velocities are calculated by $\langle v\rangle=\int \rho v d z / \int \rho d z$ with $v=v_{x}$ or $v_{y}$. The left column shows surface density, and the middle and the right columns show $\left\langle v_{x}\right\rangle,\left\langle v_{y}\right\rangle$, respectively. At early stages, only scattered high surface density spots appear. The large-scale spatial correlation of these overdense regions is evident, however, even at early times. The mean velocities also have small amplitudes at early stages. The large-scale converging (in-plane) velocity regions that eventually lead to the most prominent filaments are already evident from the first frames, however. At late stages, the overdense regions start to collect into filaments. The converging (in-plane) velocities grow due to self-gravity of the forming filaments; in addition, purely hydrodynamic instabilities (such as the nonlinear thin-shell instability, e.g., Vishniac 1994; Heitsch et al. 2007) in the shock-bounded layer may enhance early growth of perturbations. ${ }^{3}$ When converging in-plane flows become supersonic, discontinuities in the density and velocity

\footnotetext{
3 We have conducted comparison tests of selected models without self-gravity, finding that surface density fluctuations can grow to order-unity level in high Mach number cases.
} 

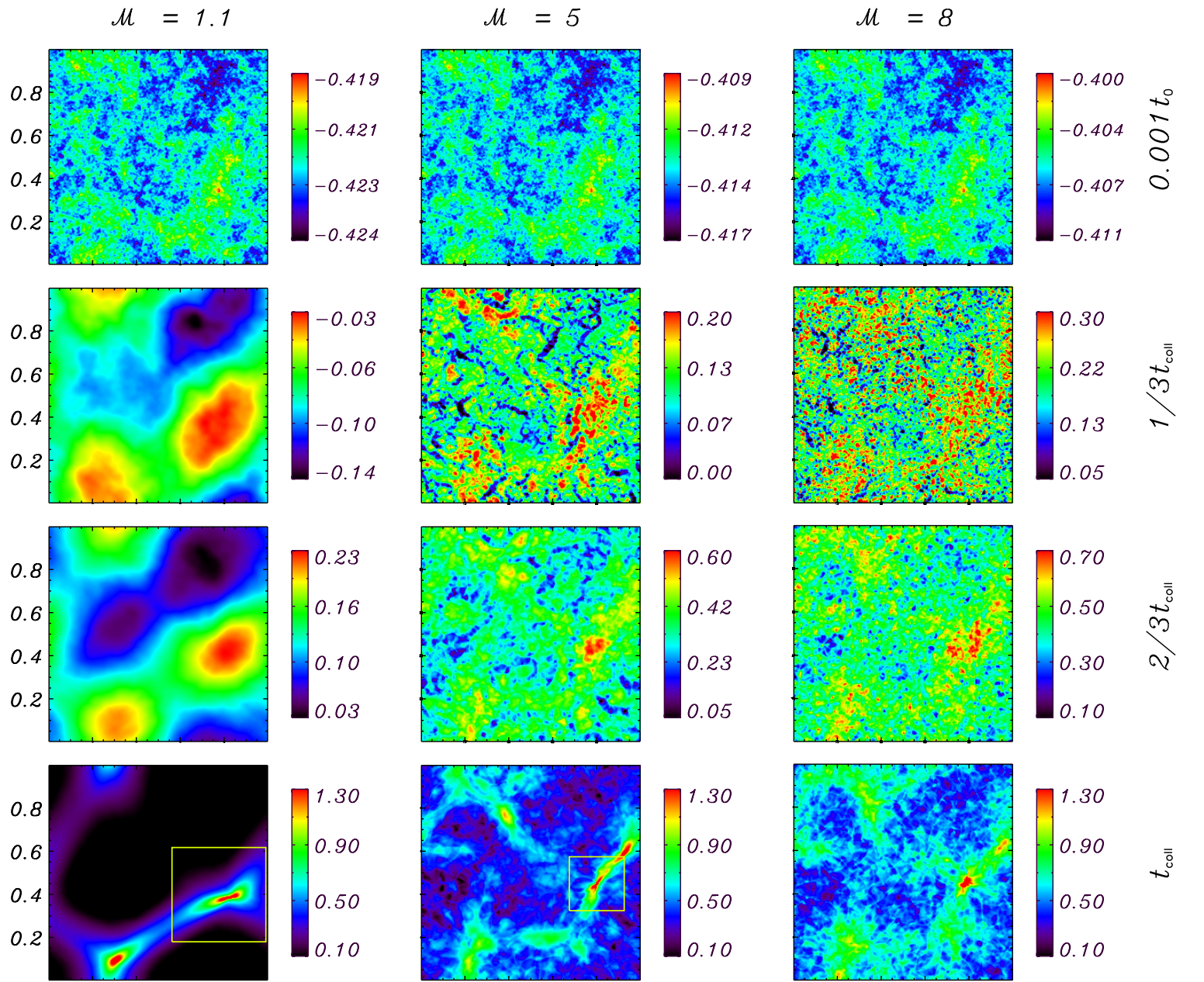

$\begin{array}{lllll}0.2 & 0.4 & 0.6 & 0.8\end{array}$
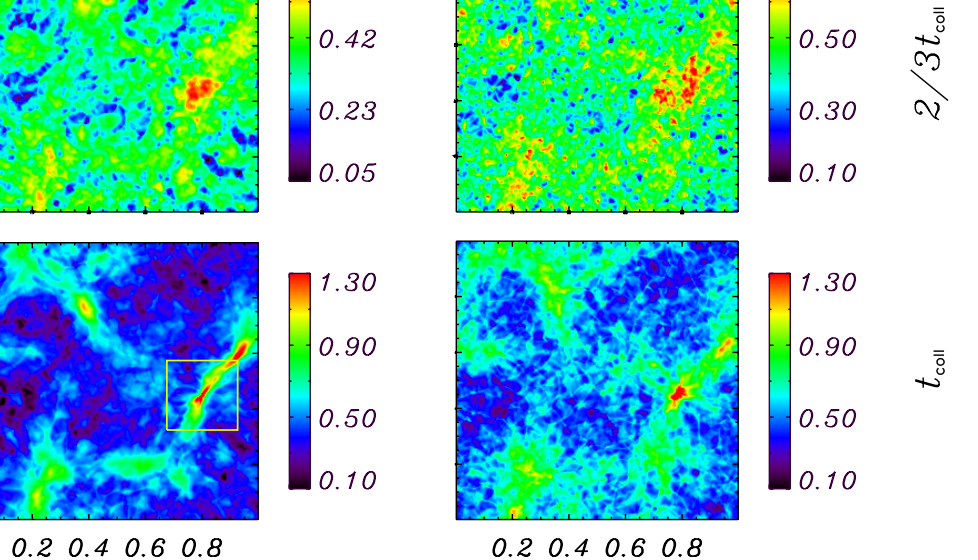

Figure 1. Evolution of surface density projected in the z-direction (color scale $\log \Sigma / \Sigma_{0}=\log N / N_{0}$; see Equations (36) and (37)) for converging-flow Mach number $\mathcal{M}=1.1$ (left column), $\mathcal{M}=5$ (middle column) and $\mathcal{M}=8$ (right column) models with the same initial perturbation patterns. The four panels from top to bottom in the each column show surface density snapshots at four instants: $t=0.001 t_{0}, 1 / 3 t_{\text {coll }}, 2 / 3 t_{\text {coll }}$, and $t_{\text {coll }}$, with $t_{\text {coll }}$ the duration of the whole simulation. These three simulations have $10 \%$ initial perturbation amplitude (see Equation (43)). The values of $t_{\text {coll }}$ are $0.636 t_{0}, 0.280 t_{0}$, and $0.232 t_{0}$ for $\mathcal{M}=1.1,5$, and 8 , respectively (see Equation (6) for definition of $t_{0}$ ). Cores are clearly smaller and more irregular for high- $\mathcal{M}$ models. The squares indicate the most evolved cores for $\mathcal{M}=1.1$ and 5 .

(A color version of this figure is available in the online journal.)

develop. These sharp fronts, as well as the collapsing motions centered on the most evolved cores, are evident in Figure 2 at $t=11 / 12 t_{\text {coll }}, t_{\text {coll }}$.

Thus, we see that turbulent motions even at sub-parsec scales seed the growth of structures, and self-gravity reinforces and amplifies these motions. The growth of dense cores and larger-scale filaments is simultaneous, both a consequence of turbulence and self-gravity.

Similar to our results in Gong \& Ostriker (2009) for spherical symmetry, we find that core building lasts most of the time up to $t_{\text {coll }}$, while the core collapse itself is rapid for the most evolved cores. Defining the "supercritical" period as the stage at which $\rho_{\text {center }} / \rho_{\text {edge }} \geqslant 10$ for the most evolved core, this first occurs at $0.589 t_{0}, 0.240 t_{0}$, and $0.209 t_{0}$, respectively, for the $\mathcal{M}=1.1,5$, and 8 models shown in Figure 1 (we note that $\rho_{\text {edge }}$ is close to the post-shock density). Taking the difference with $t_{\text {coll }}, \Delta t_{\text {supcrit }} / t_{0}=0.047,0.040$, and 0.023. From Gong \& Ostriker (2009), the supercritical stage lasts about $10 \%$ of $t_{\text {coll }}$ for cores found in shocked converging spherical flows. For the three cases shown here, $\Delta t_{\text {supcrit }} / t_{\text {coll }}$ is $7 \%, 14 \%$, and $10 \%$, consistent with our previous results. The core building stage lasts about $90 \%$ of $t_{\text {coll }}$.
To express $\Delta t_{\text {supcrit }}$ in terms of observables, we renormalize using the mean core density $\rho_{\text {mean }}$ at the instant of collapse. This quantity, $\Delta t_{\text {supcrit }} / t_{\text {ff }}\left(\rho_{\text {mean }}\right)=\Delta t_{\text {supcrit }} / t_{0} \times 3.27\left(\rho_{\text {mean }} / \rho_{0}\right)^{1 / 2}$ is measured to be $0.9,2.1$, and 0.8 for $\mathcal{M}=1.1,5$, and 8 , respectively; i.e., $\Delta t_{\text {supcrit }}$ is comparable to $t_{\text {ff }}\left(\rho_{\text {mean }}\right)$. The values of $\Delta t_{\text {supcrit }}$ are $6.6 \times 10^{5} \mathrm{yr}, 5.6 \times 10^{5} \mathrm{yr}$, and $3.2 \times$ $10^{5} \mathrm{yr}$ for $\mathcal{M}=1.1,5$, and 8 , respectively, if we take the inflowing ambient medium density as $n_{\mathrm{H}, 0}=100 \mathrm{~cm}^{-3}$; these are reduced to $2 \times 10^{5} \mathrm{yr}, 1.7 \times 10^{5} \mathrm{yr}$, and $1 \times 10^{5} \mathrm{yr}$ for $n_{\mathrm{H}, 0}=$ $1000 \mathrm{~cm}^{-3}$.

Figure 3 shows the cross-sections of the density and velocity field across the center of the most evolved cores (the locations of these cores are indicated in Figure 1) for $\mathcal{M}=1.1,5$ during the late collapse phase. The instants of the plot for $\mathcal{M}=1.1,5$ are $0.625 t_{0}$ and $0.273 t_{0}$, respectively. The top panels show the $x-y$ cross-section of density and velocity vectors composed of $v_{x}$ and $v_{y}$ in the same plane. The bottom part shows the $x-z$ cross-section and velocity vectors composed of $v_{x}$ and $v_{z}$. The velocity field clearly shows inward collapse. The amplitudes of the velocity field are smaller in the outer part and larger in the inner part, indicating the core is at a very late stage of the "outside-in" collapse. 

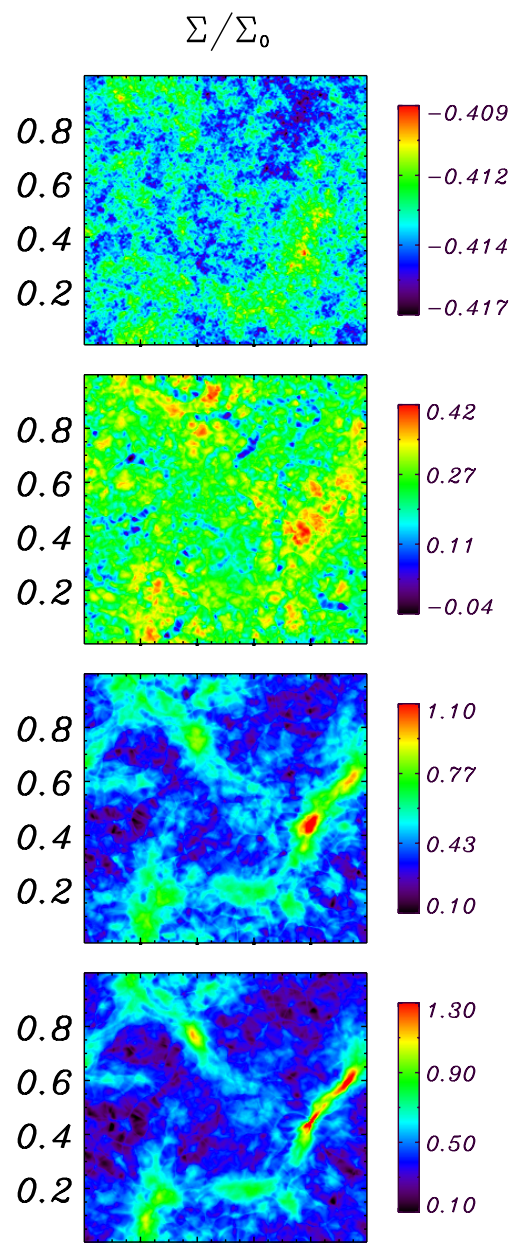

0.20 .40 .60 .8
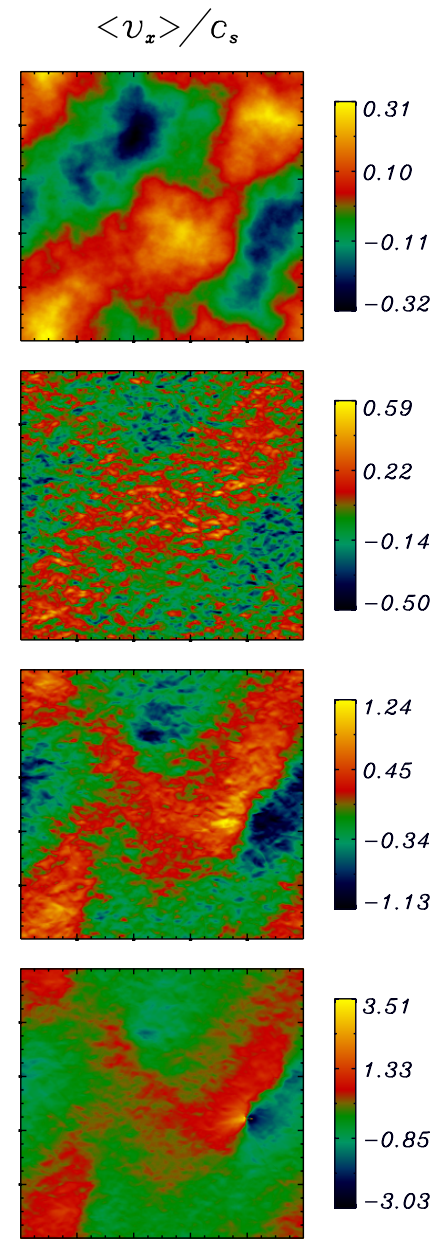

0.20 .40 .60 .8
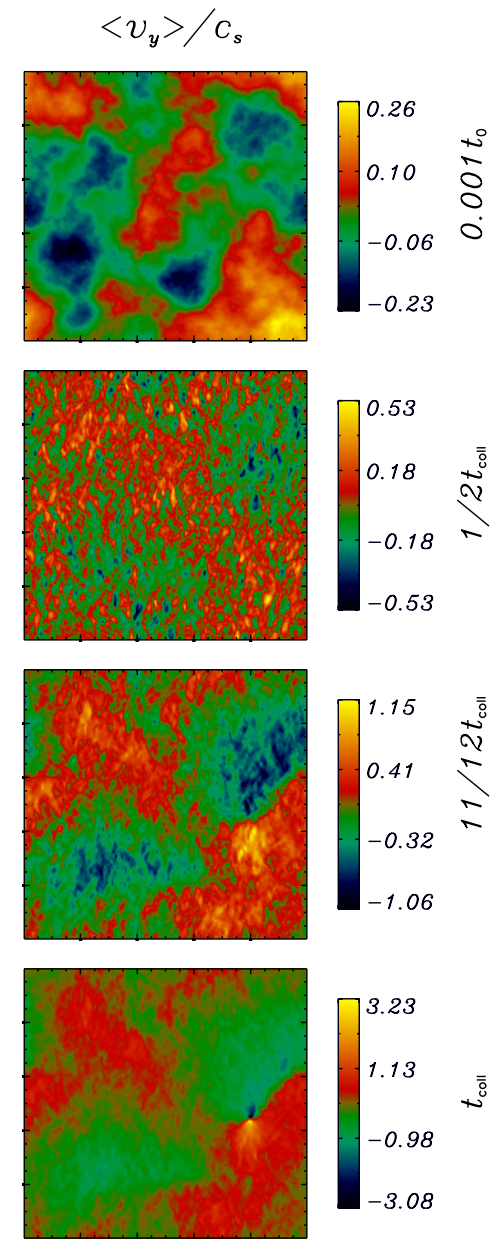

0.20 .40 .60 .8

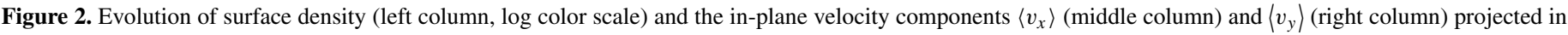

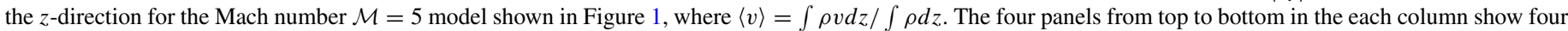

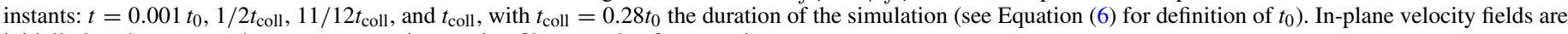
initially low, but grow to become supersonic, creating filaments that fragment into cores.

(A color version of this figure is available in the online journal.)

Figures 4 and 5 show the evolution of the density and velocity profiles of the cores in Figure 3. The density profiles are azimuthally averaged over the $x-y$ plane. The velocity profiles are along each cardinal axis $(\hat{x}, \hat{y}, \hat{z})$ through the core center. The instants for the four profiles have equal intervals $0.027 t_{0}$ for $\mathcal{M}=1.1$ and equal intervals $0.019 t_{0}$ for $\mathcal{M}=5$, respectively. The first instant for both cases is subcritical (i.e., $\left.\rho_{\text {center }} / \rho_{\text {edge }} \leqslant 10\right)$ and the second instant is close to $t_{\text {supcrit }}$. The dramatic increase of the central density during collapse is clearly evident for both cases, and the collapse develops in an "outside-in" manner with the maximum in $v$ moving inward in time. The density profile approaches the asymptotic "Larson-Penston" profile $\rho / \rho_{0}=8.86\left(r / L_{J}\right)^{-2} /(2 \pi)^{2}$ at the instant of central singularity formation, and the in-plane velocities $v_{x}, v_{y}$ approach $-3.3 c_{s}$, which is the "Larson-Penston" limit. Before the time $t_{\text {supcrit }}$ is reached, the velocity is subsonic throughout the core region. For all of the simulations we have conducted, the peak of the velocity profile becomes supersonic only at the very end of the collapse stage, similar to the results shown here.

Overall, we conclude that the evolution of individual cores in these 3D simulations follows a similar progression to the spherically symmetric 1D simulations of Gong \& Ostriker
(2009). The core building stage lasts over $90 \%$ of the time to collapse, and cores become more stratified over time. The onset of the collapse is in an "outside-in" manner, and leads to a dramatic increase in the central density. As a central singularity is approached, the density and velocity profiles approach the "Larson-Penston" asymptotic solution. These cores form and collapse within larger-scale filaments that also grow in contrast over time.

\section{CORE-FINDING METHOD}

The algorithm adopted for core-finding can either subtly or more seriously affect the core properties that result (e.g., Pineda et al. 2009). The most commonly used methods in observational work are based on contouring column density or emission intensity (e.g., the popular Clumpfind method of Williams et al. 1994). For theoretical work, density-contouring methods, sometimes incorporating further tests to determine if a structure is gravitationally bound, have frequently been used (e.g., Gammie et al. 2003). Here we shall instead use the gravitational potential isosurfaces to identify cores. In very recent work, Smith et al. (2009) took a similar approach, noting that one advantage of the gravitational potential is that 

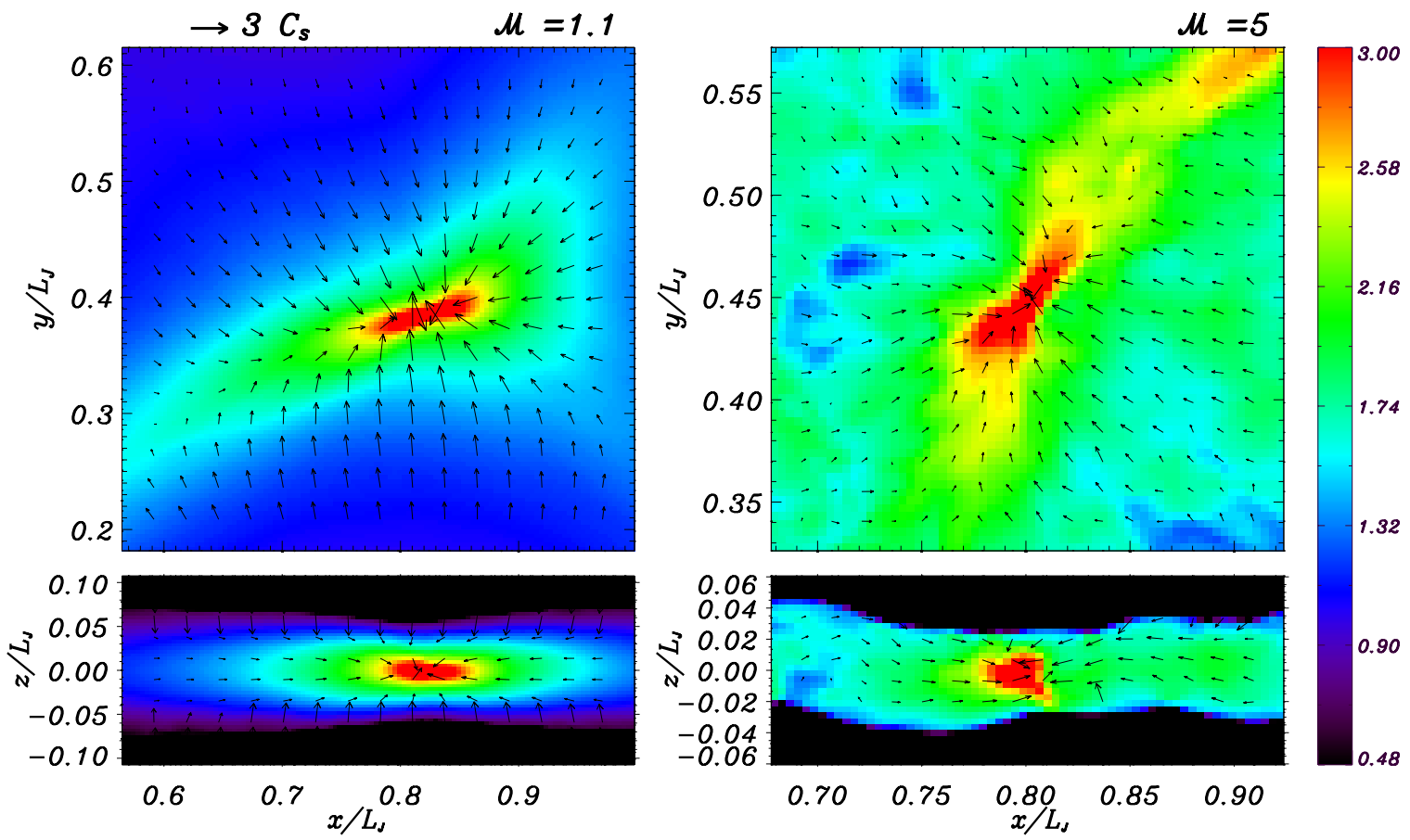

Figure 3. Density and velocity field cross-sections at the time $t_{\text {coll }}$ in the most evolved core, for $\mathcal{M}=1.1$ (left column) and $\mathcal{M}=5$ (right column). These correspond to the most evolved cores (as indicated with boxes) in Figure 1 for $\mathcal{M}=1.1,5$, respectively. The color scale represents $x-y$ and $x-z$ slices through the volume density $\left(\log \rho / \rho_{0}\right)$. The direction and length of arrows indicate the direction and magnitude of the local velocity, with scale as indicated in the upper left. At this stage of collapse, velocities increase toward the center.

(A color version of this figure is available in the online journal.)
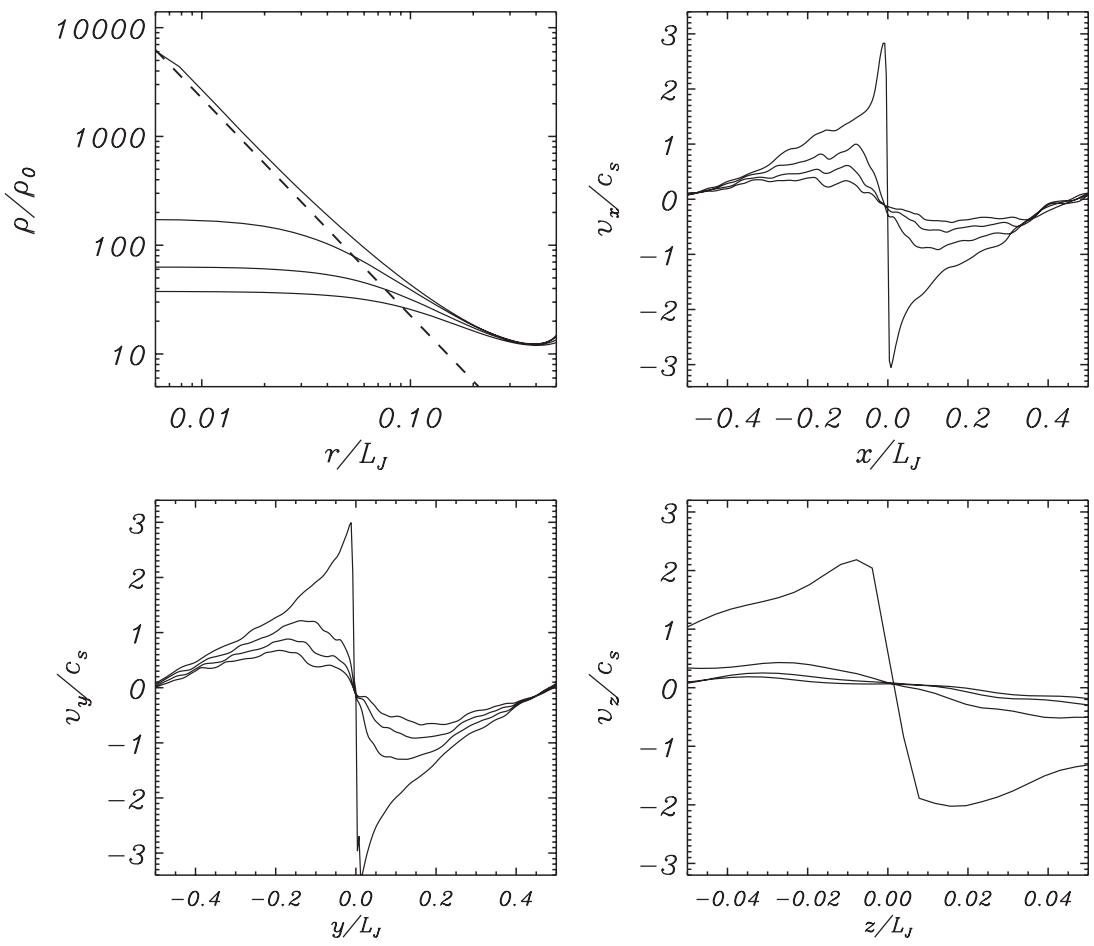

Figure 4. Radial density and velocity profiles during collapse, for the most evolved core shown in Figures 1 and 3 for $\mathcal{M}=1.1$. The density profiles are averaged azimuthally in the $x-y$ plane about the center of the core. The dashed line is the Larson-Penston asymptotic density profile $\rho / \rho_{0}=8.86\left(r / L_{J}\right)^{-2} /(2 \pi)^{2}$ (i.e., $\left.\rho=8.86 c_{s}^{2} /\left[4 \pi G r^{2}\right]\right)$. The other three plots show the corresponding velocity profiles versus distance in the $x$-, $y$-, and $z$-direction, respectively. The instants shown are $0.549 t_{0}, 0.576 t_{0}, 0.603 t_{0}, 0.632 t_{0} \approx t_{\text {coll }}$, with the most evolved profiles in each case having the largest excursions. The collapse develops in an "outside-in" manner with the maximum in $v$ moving inward with time. The density profile approaches the Larson-Penston profile with time.

it yields smoother core boundaries than the density. Another advantage is that the gravitational potential connects more directly to the fundamental physics that determines core evolu- tion. During formation stages, self-gravity gathers material to build up cores, and later it drives the collapse of supercritical cores. 

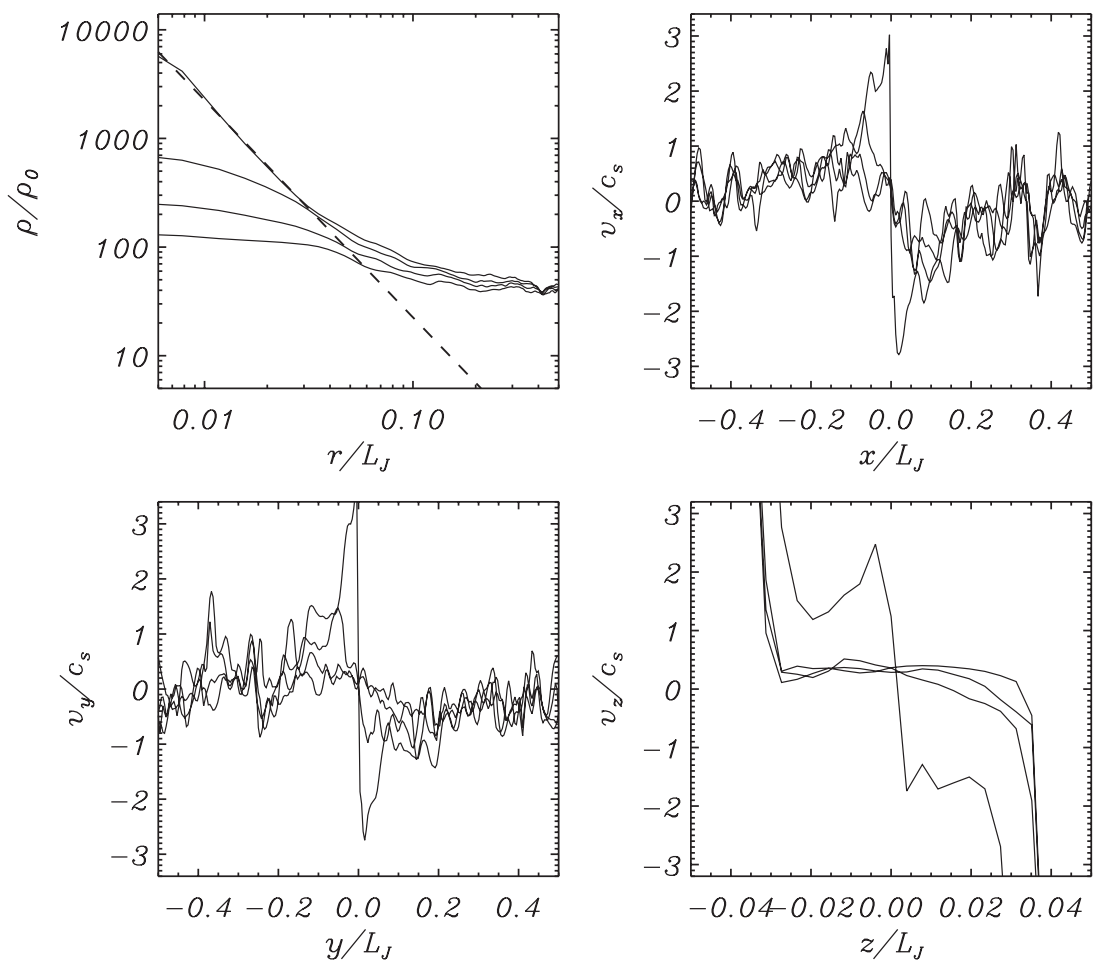

Figure 5. Same as in Figure 4 for the most-evolved core of the $\mathcal{M}=5$ model shown in Figure 1 . The profiles are shown at $t=0.219 t_{0}, 0.238 t_{0}, 0.257 t_{0}, 0.276 t_{0}$, with the density at the final time reaching the Larson-Penston solution.

To identify cores via the gravitational potential, we first find and mark all the local minima of the gravitational potential; second, we find the largest closed potential contour (or isosurface) surrounding each individual minimum. In the second step, we increase the contour level from the bottom of a given potential well step by step until it violates another minimum's marked territory. We define the region enclosed by the largest closed contour as a core. The contour interval $\Delta \Phi$ has negligible effect on the results as long as it is small enough (typically $\leqslant 0.03 c_{s}^{2}$ ). If the distance between two minima is smaller than 10 pixels (corresponding to a physical distance $\sim 0.03-0.1 \mathrm{pc}$ for $n_{\mathrm{H}, 0} \sim 10^{2}-10^{3} \mathrm{~cm}^{-3}$ ), the regions associated with these two minima are merged and treated as a single core. Since we do not continue the simulation after the most evolved core collapses, we apply the algorithm to the last output from each simulation.

Since gas with sufficient thermal and kinetic energy need not be permanently (or even temporarily) bound to a given core, the gravitational potential is not the final word. The lower density outer parts of a core are the most subject to loss. We can test this effect on core identification by adding thermal energy to the gravitational energy, and only assigning a given fluid element to a core if $E_{\mathrm{th}}+E_{g}<0$. For any fluid element, the specific thermal energy is taken to be $E_{\mathrm{th}}=3 / 2 c_{s}^{2}$, and the specific gravitational potential energy is taken to be $E_{g}=\Phi-\Phi_{\max }$, where $\Phi_{\max }$ is the potential of the largest closed contour that defines the core. ${ }^{4}$ Including a thermal energy condition in core definition decreases the volume (or area in 2D) of the cores. Of course, the thermal energy can in fact be radiated away,

\footnotetext{
4 We note that $\left|E_{g}\right|$ for a core embedded within a dense filament (or sheet) may be much lower than $\left|E_{g}\right|$ for the same core in isolation. In assessing whether a core is bound, it is crucial to take tidal gravity effects into account. If these tidal effects are neglected, $\left|E_{g}\right|$ will be overestimated by a factor $\sim \Sigma_{\text {core }} /\left(\Sigma_{\text {core }}-\Sigma_{\text {filament }}\right)$, which is quite large if the contrast between a core and its surroundings is modest.
}

so that gas that is initially near the largest closed contour may become more strongly bound after the interior of a core collapses. In this case, the potential alone could determine the final core mass. Short of following cores through the final stages of star formation, we consider it useful to compare cores with and without a thermal-gravitational energy criterion. Hereafter, we term our core-finding method "gravitational identification" (GRID). We refer to the region within the largest closed gravitational potential isosurface surrounding each local minimum as a GRID-core. For each GRID-core, the region which has $E_{\mathrm{th}}+E_{g}<0$ is referred to as a bound GRID-core.

Because volume density data cubes are not directly accessible in observations, three-dimensional gravitational potential contouring is only applicable to model data from numerical simulations. It is therefore interesting to explore gravitational potential contouring of surface density maps, which are direct observables. To identify cores in a surface density map, we have to calculate the gravitational potential first. For a layer of half-thickness $H$, the gravitational potential component $\Phi_{k, 2 \mathrm{D}}$ of surface density component $\Sigma_{k}$ (Fourier transform of Equation (36)) in phase space is

$$
\Phi_{k, 2 \mathrm{D}}=-\frac{2 \pi G \Sigma_{k}}{|\boldsymbol{k}|(1+|k H|)},
$$

where $|\boldsymbol{k}|=\sqrt{k_{x}^{2}+k_{y}^{2}}$. Note that for $|\boldsymbol{k} H| \gg 1, \Phi_{\boldsymbol{k}, 2 \mathrm{D}} \sim$ $-4 \pi G \rho_{k} / k^{2}$, which is the solution of the Poisson equation in three dimensions, for $\rho_{k}=\Sigma_{k} / 2 H$. For $|k H| \ll 1$, Equation (45) is the solution of the Poisson equation for an infinitesimally thin layer. The gravitational potential $\Phi_{2 \mathrm{D}}(x, y)$ is the inverse Fourier transform of $\Phi_{k, 2 D}$. Given the 2D gravitational potential field $\Phi_{2 \mathrm{D}}(x, y)$, we can apply the GRID procedure as for 3D. In Section 6, we will compare the results from GRID using $\Phi(x, y, z)$ and $\Phi_{2 \mathrm{D}}(x, y)$ (using $H=\delta z$ ). Hereafter, we use " $2 \mathrm{D}$ " to denote the results from applying the 

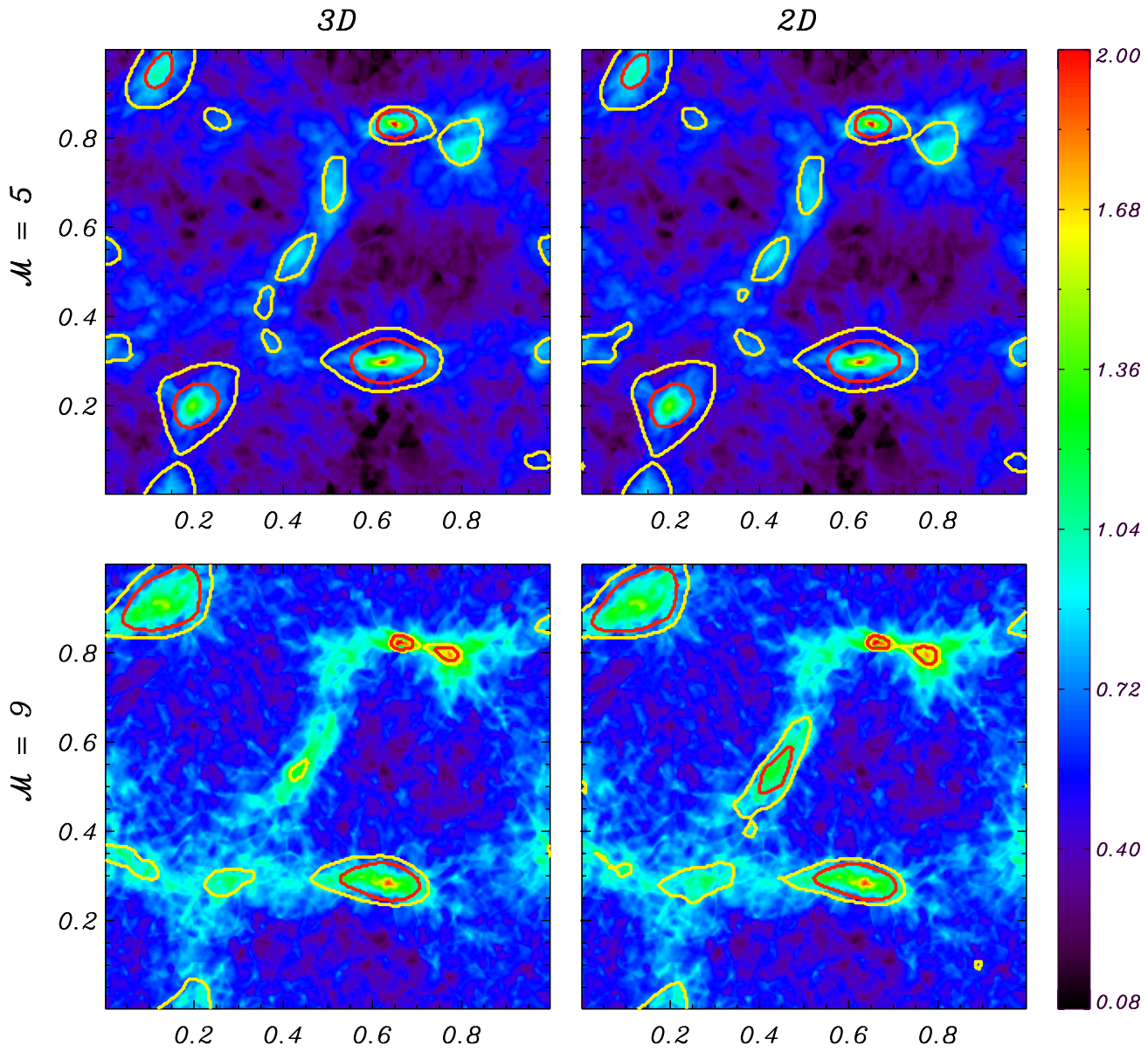

Figure 6. Comparison of GRID-cores defined via the gravitational potential computed from 3D volume density ( $\Phi$, left column) and $2 \mathrm{D}$ projected surface density ( $\Phi_{2 \mathrm{D}}$, right column). The top row shows $\mathcal{M}=5$ and bottom row $\mathcal{M}=9$. The areas enclosed by yellow curves are the GRID-cores determined by the largest closed gravitational potential ( $\Phi$ or $\Phi_{2 \mathrm{D}}$ ) contour surrounding a local potential minimum, and the areas enclosed by red curves are the bound GRID-cores. Color scale shows projected surface density $\left(\log \Sigma / \Sigma_{0}\right)$ in all panels. Cores identified using $\Phi$ and $\Phi_{2 \mathrm{D}}$ agree quite well.

(A color version of this figure is available in the online journal.)

GRID method to surface density and "3D" for applying the GRID method to the volume density.

As an example, Figure 6 shows the comparison of GRIDcores and bound GRID-cores between 3D and 2D for $\mathcal{M}=5$ and 9. The top portion shows core areas identified for the $\mathcal{M}=5$ model using $\Phi$ (top left) and $\Phi_{2 \mathrm{D}}$ (top right). The bottom portion shows the same comparison for $\mathcal{M}=9$ with cores found from $\Phi$ (bottom left) and from $\Phi_{2 \mathrm{D}}$ (bottom right). (Note that the $\mathcal{M}=5$ and $\mathcal{M}=9$ simulations have the same initial velocity perturbations patterns, which is why the overall structure is similar.) In all plots, the areas enclosed by yellow contours are the GRID-cores and the areas enclosed by red contours are the bound GRID-cores. The core areas for the 3D plots are the projection of the 3D core volume onto the $z=0$ plane. For the $\mathcal{M}=5$ model, the $2 \mathrm{D}$ and $3 \mathrm{D}$ core-finding procedures identify 12 and 13 cores, respectively; the cores and the bound regions are located at nearly the same positions. For the $\mathcal{M}=9$ model, seven cores are identified for both cases. One bound core in 2D lacks a 3D counterpart, implying the corresponding potential well in 3D is too shallow (see discussion of potential well depths in Section 6).

In addition to finding almost all of the same core centers (defined by the potential minimum), the areas marked by the 3D and 2D GRID algorithms are almost the same. Figure 7 shows the results of GRID for four simulations for $\mathcal{M}=5$. The white contours mark GRID-cores from 3D density and the green contours mark GRID-cores from 2D surface density. The red and yellow contours mark the bound GRID-cores for 3D and 2D respectively. The areas identified for the cores agree quite well. Over all, we conclude that the 2D GRID algorithm can give nearly identical core-finding areas as the 3D GRID algorithm.

In spite of the overall similarity between 2D and 3D GRIDcore finding, there are minor differences in the results. In the each panel of Figure 7, a few GRID-cores in relatively lowdensity regions are identified in 2D but not in 3D. In comparing core properties between 2D and 3D, we shall apply additional resolution criteria to eliminate these small, shallow cores.

\section{CORE PROPERTIES}

To obtain a sufficient statistical sample, we conduct 20 simulations for each value of the Mach number $(\mathcal{M}=$ $1.1,2,3,4,5,6,7,8,9)$ and compute GRID-core masses and radii for each model (180 models total). Each of the 20 simulations for a given $\mathcal{M}$ is perturbed by a different realization of the velocity field. As an example of the differences with different random realizations of the power spectrum, Figure 7 shows the snapshots of surface density at a late stage for four different $\mathcal{M}=5$ simulations. The 3D GRID-core numbers are 9, 6, 9, and 7 . The corresponding core mass ranges are [0.00151, 0.158] $M_{0},[0.0051,0.128] M_{0},[0.0013,0.242] M_{0}$, and $[0.031,0.250]$ $M_{0}$. The core numbers and core masses from simulations with 


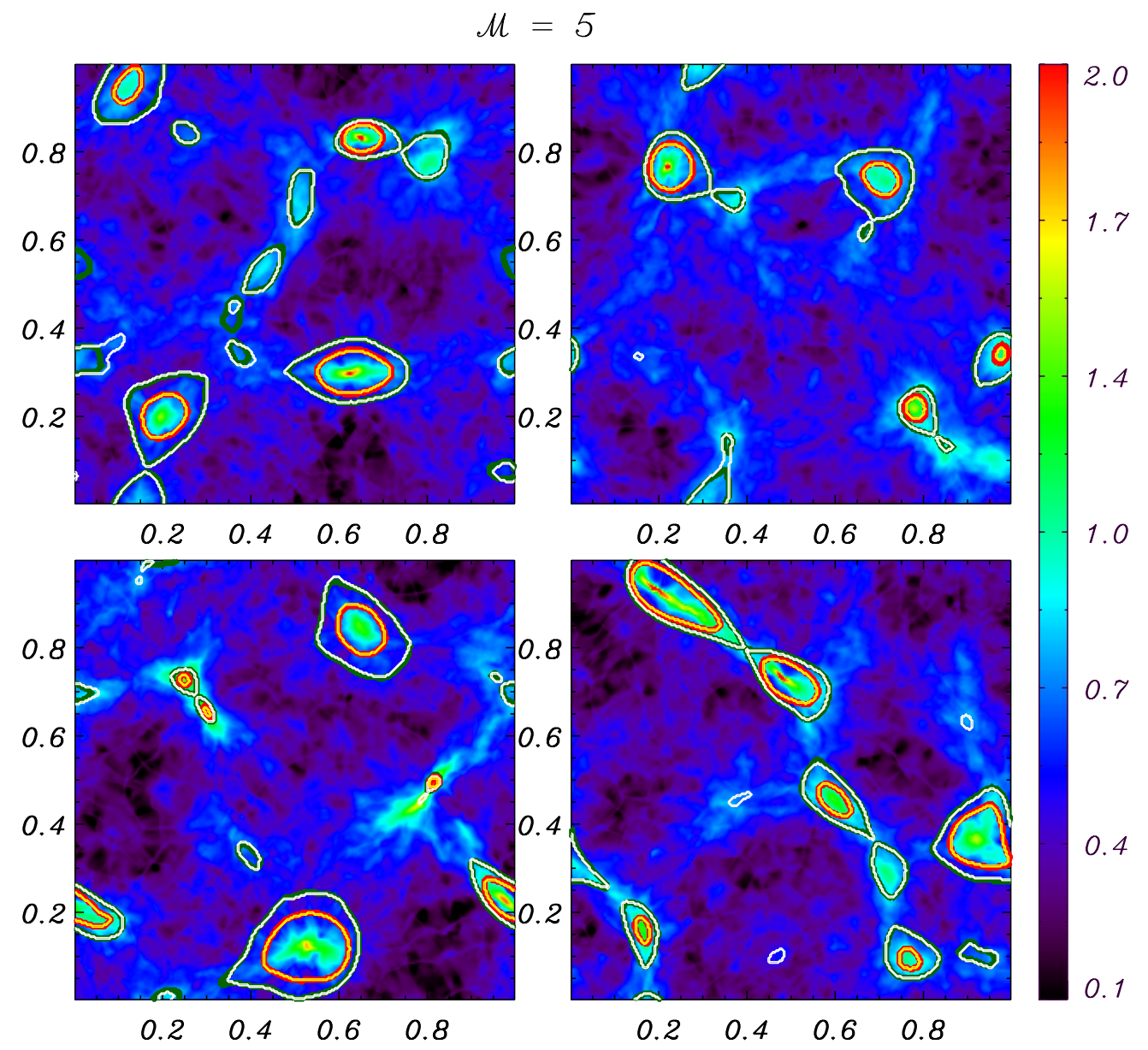

Figure 7. Late-stage surface density $\left(\log \Sigma / \Sigma_{0}\right)$ and GRID-core comparison for four different random perturbation realizations of the $\mathcal{M}=5$ model. The snapshots are at $t=0.282 t_{0}, 0.304 t_{0}, 0.304 t_{0}, 0.302 t_{0}$ from left to right and top to bottom. The corresponding maximum densities are $1.0 \times 10^{5} \rho_{0}, 1.53 \times 10^{5} \rho_{0}, 8.18 \times$ $10^{4} \rho_{0}, 1.34 \times 10^{5} \rho_{0}$. The white and green curves are GRID-cores defined by the largest closed contour of the gravitational potential $\left(\Phi\right.$ and $\Phi_{2 \mathrm{D}}$, respectively) surrounding each potential minimum. The red and yellow curves are the bound GRID-cores obtained using $\Phi$ and $\Phi_{2 \mathrm{D}}$, respectively. Except for a few small, shallow cores, the core-finding algorithms in $2 \mathrm{D}$ and $3 \mathrm{D}$ give quite similar results.

(A color version of this figure is available in the online journal.)

different seeds are in a similar range; the same is true for cases with other Mach numbers.

The GRID-core masses for $3 \mathrm{D}$ and $2 \mathrm{D}$ are $M_{3 \mathrm{D}}=$ $\int \rho d x d y d z$ and $M_{2 \mathrm{D}}=\int \Sigma d x d y$, respectively. The GRIDcore radius for $3 \mathrm{D}$ is defined as the equivalent radius of a $3 \mathrm{D}$ sphere with the same volume $V_{3 \mathrm{D}}: r_{3 \mathrm{D}} \equiv\left(3 V_{3 \mathrm{D}} / 4 \pi\right)^{1 / 3}$. The effective 2D GRID-core radius is calculated from the area $S_{2 \mathrm{D}}$ of the core region as: $r_{2 \mathrm{D}} \equiv\left(S_{2 \mathrm{D}} / \pi\right)^{1 / 2}$. To ensure that identified GRID-cores are numerically well resolved, we only retain cores with effective radii $\geqslant 4$ zones. We define a background surface density as the mean of the bottom $10 \%$ of the surface density; this mean value can be subtracted from the surface density in the core region when calculating $M_{2 \mathrm{D}}$. As mentioned in Section 2, a more restrictive definition includes only gas with thermal plus gravitational energy negative; these bound GRID-cores are first identified by the gravitational potential, and then pixels are excluded if the sum of thermal energy and gravitational potential is greater than 0 .

Figure 8 shows $M_{2 \mathrm{D}}$ versus $M_{3 \mathrm{D}}$ for GRID-cores, for each Mach number of the low-amplitude perturbation set. Note that only cores with same center of the local potential minima are shown here. Both 2D GRID-core masses without background subtraction $\left(M_{2 \mathrm{D}}\right.$, diamonds in the figure) and 2D GRID-core masses with background subtraction $\left(M_{2 \mathrm{D}, \mathrm{bs}}\right.$, dots in the figure) are shown versus $M_{3 \mathrm{D}}$. For large masses, $M_{2 \mathrm{D}}$ agrees well with $M_{3 \mathrm{D}}$ while $M_{2 \mathrm{D} \text {,bs }}$ is slightly lower than $M_{3 \mathrm{D}}$. For small masses, $M_{2 \mathrm{D}, \text { bs }}$ agrees better than $M_{2 \mathrm{D}}$ with $M_{3 \mathrm{D}}$. Both $M_{2 \mathrm{D}}$ and $M_{2 \mathrm{D}, \mathrm{bs}}$ agree with $M_{3 \mathrm{D}}$ better for high mass than low mass.

Figure 9 shows a similar comparison of bound GRID-cores for 2D and 3D. The background surface density is subtracted for

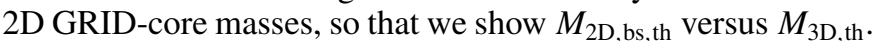
Here, the subscript "th" represents inclusion of a thermal energy criterion in defining bound GRID-cores, which eliminates most

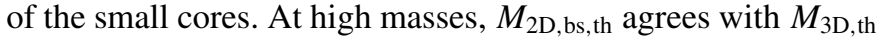

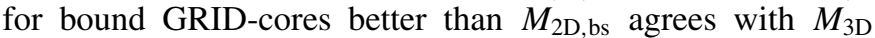
for the whole set of GRID-cores. This is because only zones sufficiently near the potential minimum where $E_{\text {th }}+E_{g}<0$ are included in bound GRID-cores; these regions are not sensitive to projection effects. At low masses, $M_{2 \mathrm{D} \text {,bs, th }}$ exceeds $M_{3 \mathrm{D}, \text { th }}$ for bound GRID-cores, meaning that imposing the thermal-gravitational energy criterion affects $M_{3 \mathrm{D} \text {,th }}$ more than $M_{2 \mathrm{D}, \mathrm{bs}, \mathrm{th}}$.

To understand the difference between the 2D and 3D GRIDcore masses, we consider the shape of the gravitational potential well for surface density and volume density. From Equation (45), $\Phi_{2 \mathrm{D}, k} \propto-k^{-1}$ whereas $\Phi_{3 \mathrm{D}, k} \propto-k^{-2}$. At larger $k$, corresponding to smaller scales, $\left|\Phi_{3 \mathrm{D}}\right|$ decreases faster than $\left|\Phi_{2 \mathrm{D}}\right|$. That means that the small 2D GRID-cores cover more 


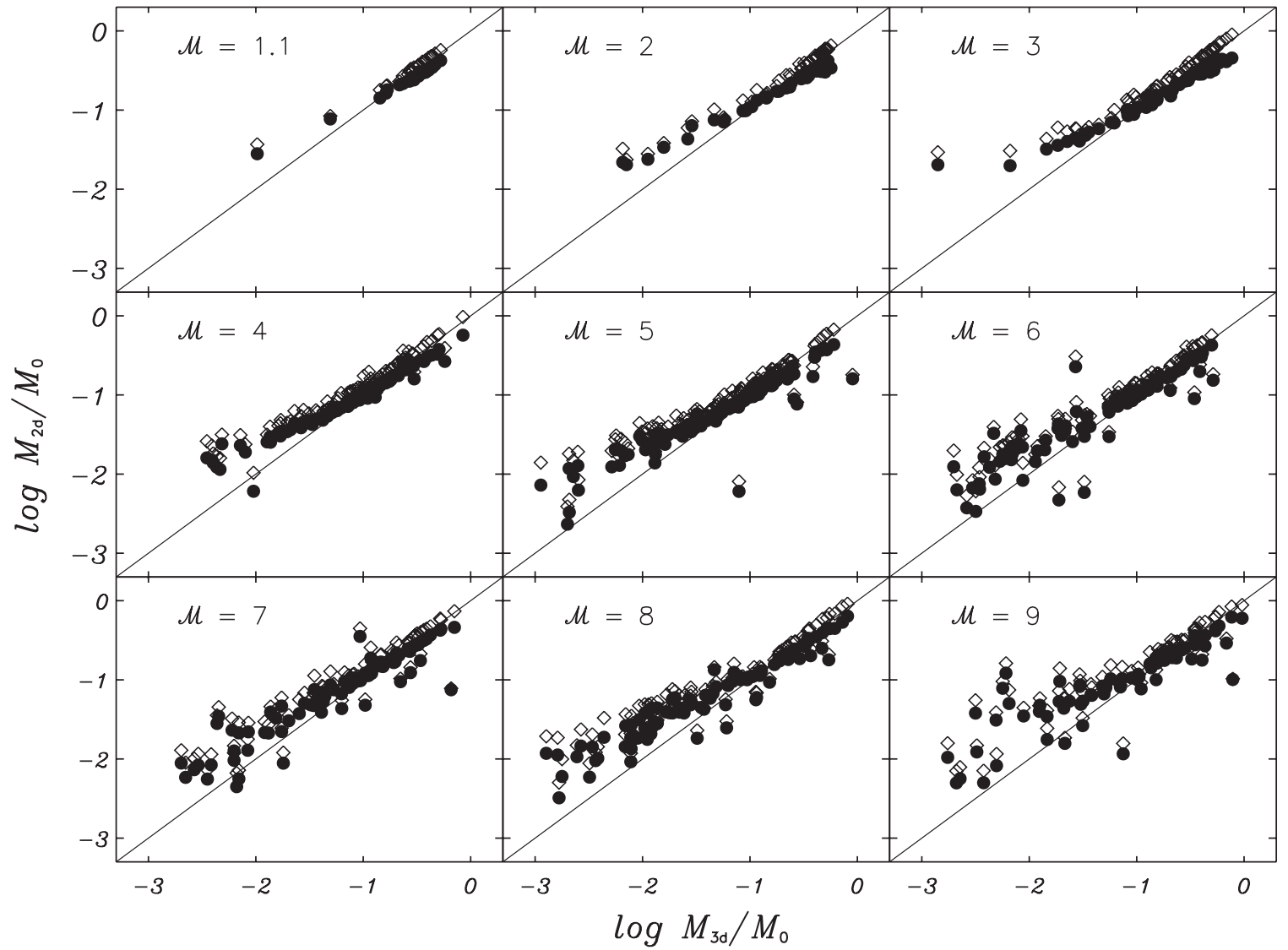

Figure 8. GRID-core mass obtained from 2D $\left(M_{2 \mathrm{D}}\right)$ vs. 3D $\left(M_{3 \mathrm{D}}\right)$. Diamonds are $M_{2 \mathrm{D}}$ for 2D GRID-cores without background subtraction, and dots are $M_{2 \mathrm{D}, \mathrm{bs}}$ for 2D GRID-cores with background subtraction. The mass unit $M_{0}$ is given in Equation (5). Solid lines represent $M_{2 \mathrm{D}}=M_{3 \mathrm{D}}$; higher-mass cores are consistent with this.

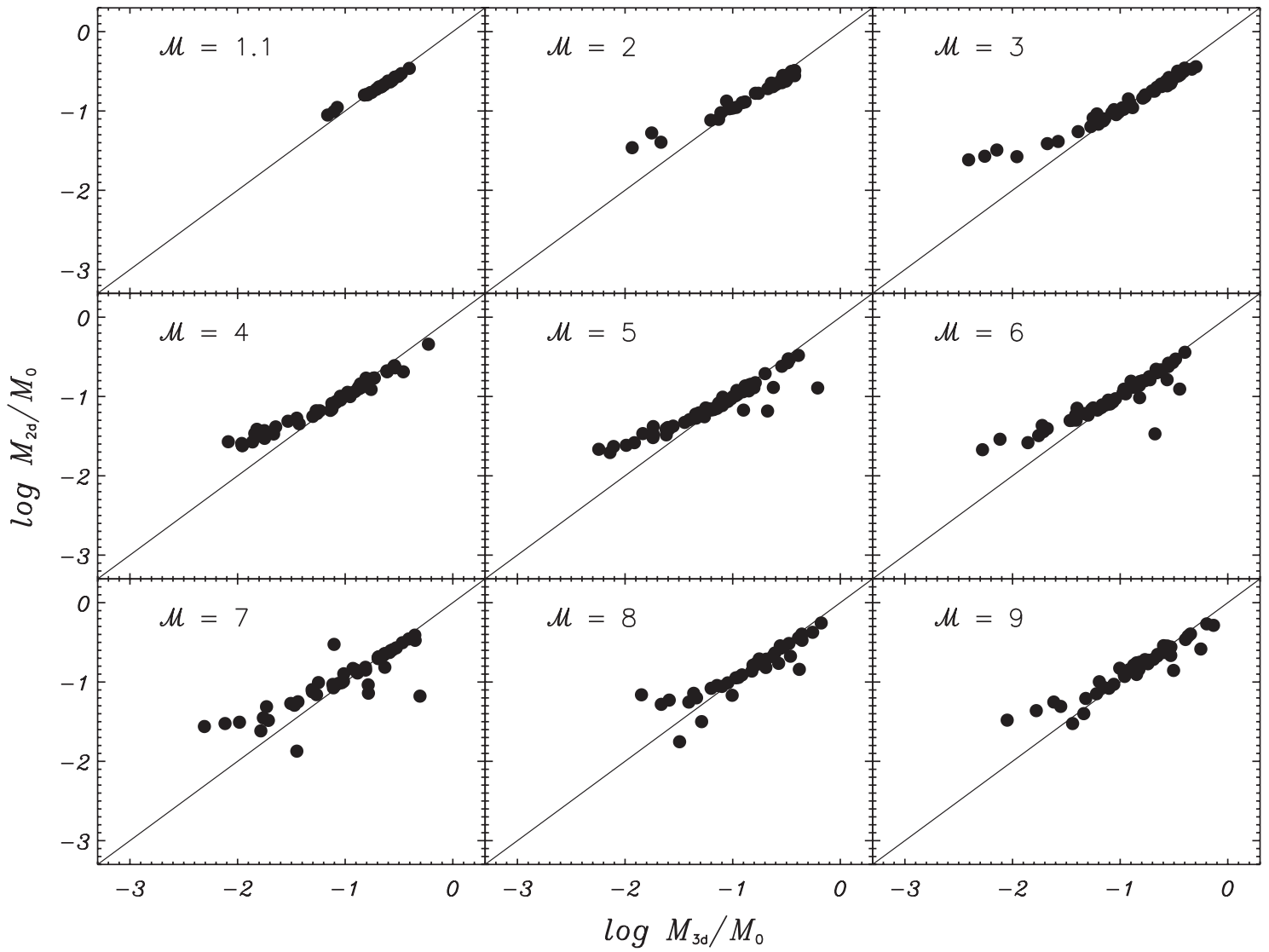

Figure 9. Bound GRID-core mass for $2 \mathrm{D}$ with background subtraction $\left(M_{2 \mathrm{D}, \mathrm{bs}, \mathrm{th}}\right)$, vs. bound GRID-core mass for $3 \mathrm{D}\left(M_{3 \mathrm{D}, \text { th }}\right)$. When the condition $E_{\mathrm{th}}+E_{g}<0$ is included in the core definition, the lowest mass cores are eliminated and $M_{2 \mathrm{D} \text {, bs, th }}$ agrees well with $M_{3 \mathrm{D} \text {,th }}$ down to $\sim 10^{-2} M_{0}$. 


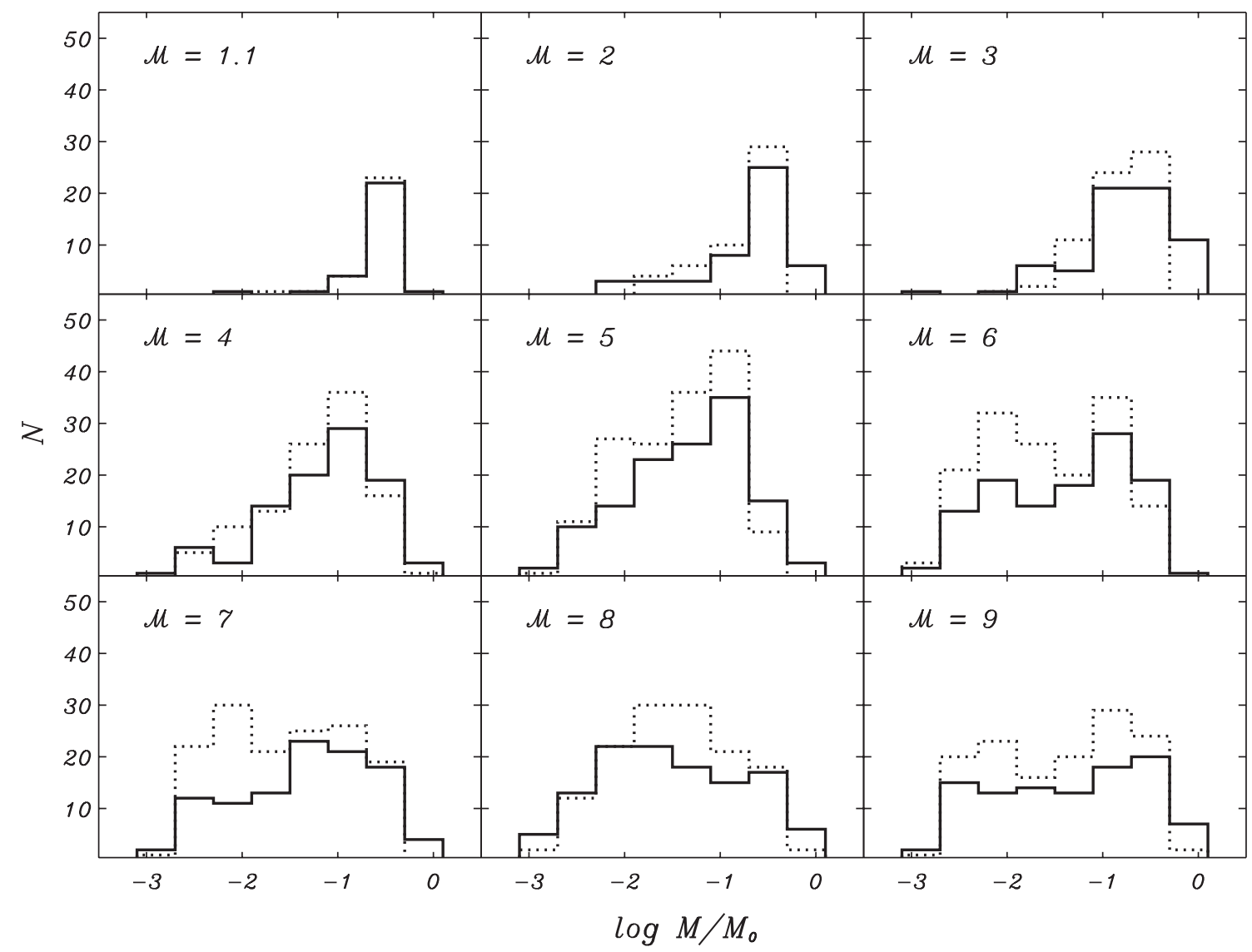

Figure 10. Histograms of all GRID-core masses found in all simulations for each Mach number $\mathcal{M}$ for low-amplitude perturbations. Solid lines are for 3D GRID-cores $\left(M_{3 \mathrm{D}}\right)$ and dashed lines are for 2D GRID-cores with background subtraction $\left(M_{2 \mathrm{D}, \mathrm{bs}}\right)$. The 2D and 3D distributions are similar for all Mach numbers.

area than small 3D GRID-cores, evident at the low end of each panel in Figure 8. Also, gravitational potential wells of middlesized 2D GRID-cores are deeper than those of 3D middle-sized GRID-cores. If the shallow parts of the potential are excluded by applying a thermal energy requirement, 3D GRID-cores are affected more than 2D GRID-cores. Moderate-mass GRID-cores that have $M_{2 \mathrm{D}, \mathrm{bs}}$ and $M_{3 \mathrm{D}}$ comparable will thus have $M_{3 \mathrm{D} \text {,th }}$ lower than $M_{2 \mathrm{D}, \mathrm{bs}, \mathrm{th}}$, as is evident in Figure 9. As mentioned in Section 5, we include the term $|\boldsymbol{k}| H$ to allow for the non-zero thickness of the layer perpendicular to the plane. This can, in principle, help decrease the gap between the 2D and 3D gravitational potentials. In practice, however, we find that the value for $H$ to make the central-to-edge value of $\Phi_{2 \mathrm{D}}$ comparable to that for $\Phi$ is smaller than $\delta z$. Although the $2 \mathrm{D}$ and 3D gravitational potentials are not exactly the same, Figure 9 shows that $2 \mathrm{D}$ and 3D bound GRID-cores masses are generally close down to $\sim 10^{-2} M_{0}$ (which is $\lesssim 1 M_{\odot}$ for typical conditions, from Equation (5)).

Figure 10 shows histograms for the distributions of $M_{2 \mathrm{D}, \mathrm{bs}}$ and $M_{3 D}$ (all GRID-cores) for each $\mathcal{M}$, while Figure 11 shows the histograms of $M_{2 \mathrm{D}, \mathrm{bs} \text {,th }}$ and $M_{3 \mathrm{D} \text {,th }}$ (bound GRID-cores), both for low perturbation amplitudes. The distributions of $M_{2 \mathrm{D}, \mathrm{bs}}$ and $M_{3 \mathrm{D}}$ are quite similar for all $\mathcal{M}$, except slightly more low-mass cores are identified for $2 \mathrm{D}$ at large $\mathcal{M}$. When the thermal-gravitational energy condition is included in defining cores, the low-mass end of the distribution is removed; in Figure 11, the 2D bound GRID-cores have almost exactly the same distributions as 3D bound GRID-cores.

Figures 12 (all GRID-cores) and 13 (bound GRID-cores) show the median core mass (squares in figures) versus $\mathcal{M}$ from
Figures 10 and 11, respectively. (We do not measure the peak because some of the histograms are irregular.) Figures 14 (all GRID-cores) and 15 (bound GRID-cores) show the same median mass $-\mathcal{M}$ relation for high-amplitude initial perturbations. The breadth of the distributions at each $\mathcal{M}$ is indicated by vertical bars: the lower bar is the difference between the median and the first quartile, and the higher bar is the difference between the third quartile and the median. In Figures 12-15, we overlay lines showing the predicted critical mass at late stages (Equation (22) or (23), dashed line with $M \propto \mathcal{M}^{-1}$ ), and the prediction for the mass that has grown the most at early time (Equation (28) or (31), dot-dashed with $M \propto \mathcal{M}^{-1 / 2}$ ). The postshock BE mass ( $M \propto \mathcal{M}^{-1}$ from Equation (7)) is similar to the late-stage critical mass.

As the Mach number increases, the post-shock density $\rho \approx$ $\rho_{0} \mathcal{M}^{2}$ is higher. This lowers the Jeans length (as well as the Jeans mass and BE mass), permitting smaller (but denser) cores to form at high $\mathcal{M}$ compared to low $\mathcal{M}$. However, high-mass cores can still form at high $\mathcal{M}$, as is evident in Figures 10 and 11 and the quartiles shown in Figures 12-15: at high $\mathcal{M}$, the histograms extend to low mass, but the high-mass part of the distribution is still present. This is consistent with the expectation that any scale above the critical scale can grow more nonlinear due to self-gravity (see Equations (12)-(17)).

Based on Figures 12-15, we also note that the median mass versus $\mathcal{M}$ relations are quite similar whether cores are identified with the $2 \mathrm{D}$ or $3 \mathrm{D}$ gravitational potential. This is true for low or high-amplitude perturbations, for both all GRID-cores and bound GRID-cores. This evidently shows that $2 \mathrm{D}$ cores have similar statistical properties to the 3D cores. Since the GRID 


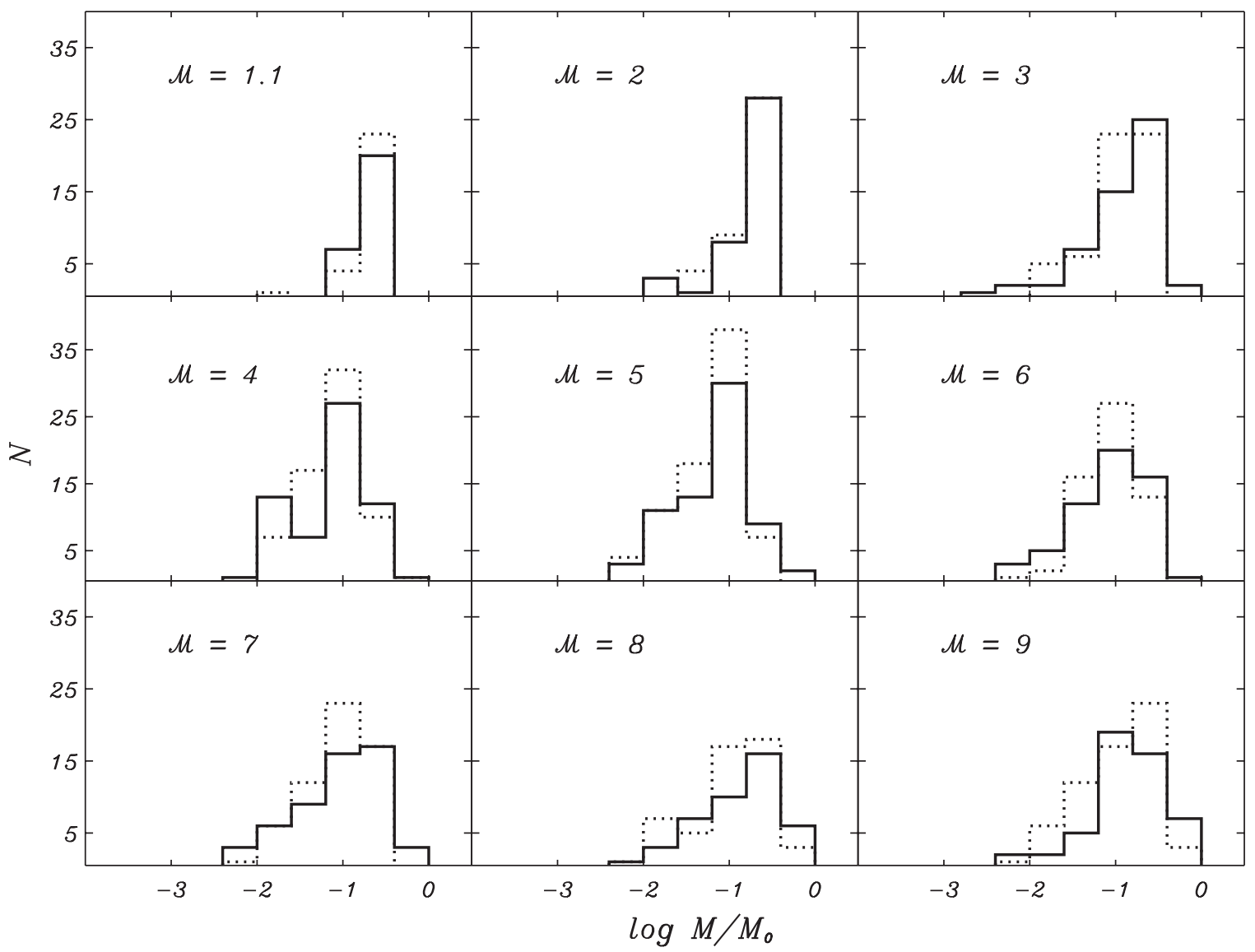

Figure 11. Same as in Figure 10, except for bound GRID-cores (i.e., mass is $M_{3 \mathrm{D} \text {,th }}$ and $M_{2 \mathrm{D}, \mathrm{bs}, \mathrm{th}}$ ). When the condition $E_{\mathrm{th}}+E_{g}<0$ is applied, most of the low-mass cores are eliminated, for every Mach number. The 2D bound GRID-cores have almost the same mass distribution as 3D bound GRID-cores.

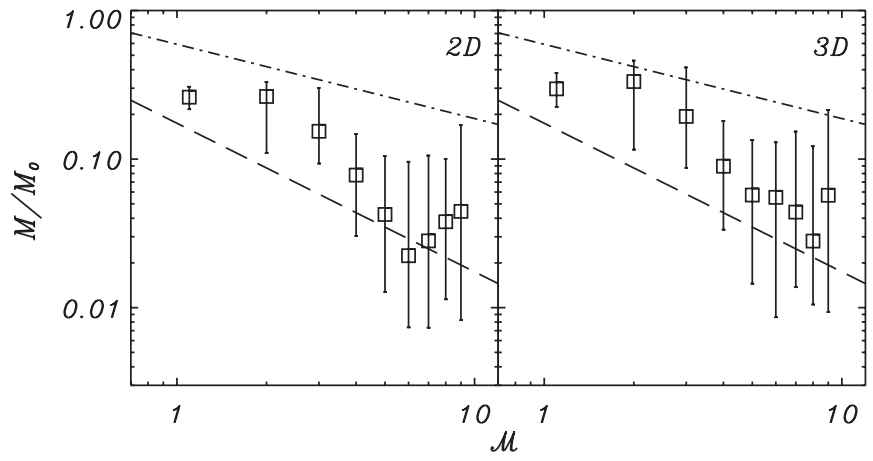

Figure 12. Median GRID-core mass $M$ vs. Mach number $\mathcal{M}$ of the inflow. The left panel is for 2D GRID-cores $\left(M_{2 \mathrm{D}, \mathrm{bs}}\right)$ and the right panel for 3D GRID-cores $\left(M_{3 \mathrm{D}}\right)$. Vertical bars indicate quartiles of the distribution. Also shown is the expected mass dependence for early gravitational fragmentation given by Equation (28) (with $M \propto \mathcal{M}^{-1 / 2}$, dot dashed), and late gravitational fragmentation given by Equation (22) (with $M \propto \mathcal{M}^{-1}$, dashed). The critical Bonnor-Ebert mass at the post-shock density (see Equation (7)) is similar to the late-stage prediction ( $M \propto \mathcal{M}^{-1}$, dashed). The relation between median core mass and $\mathcal{M}$ is quite similar for 2D and 3D cores. Core mass declines with increasing Mach number $\mathcal{M}$, lying between the $M \propto \mathcal{M}^{-1 / 2}$ (early stage) and $M \propto \mathcal{M}^{-1}$ (late stage) fragmentation predictions.

algorithm is easy to implement for observational data, it appears to be a promising method for finding cores. ${ }^{5}$

Median masses for GRID-cores decline with increasing Mach number for both low and high-amplitude perturbations (see 5 An IDL implementation of our GRID-core algorithm for use with observed
data (FITS files containing surface density maps) is available from the authors.

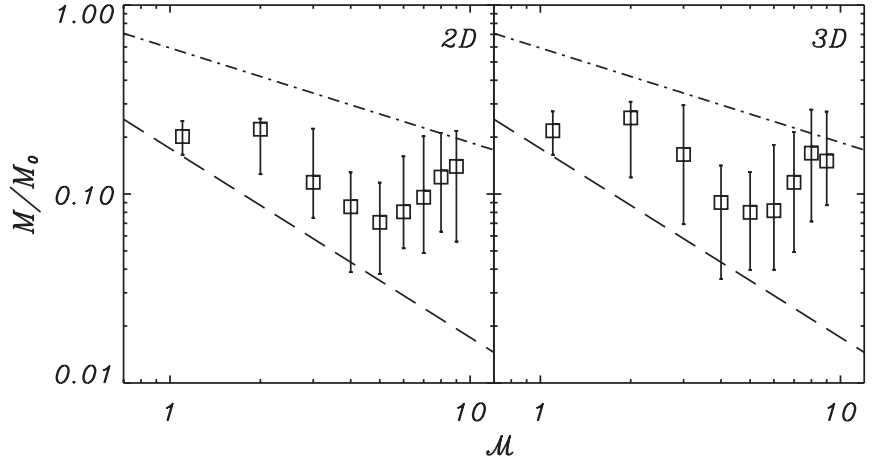

Figure 13. Same as in Figure 12, but for bound GRID-cores $\left(E_{\mathrm{th}}+E_{g}<0\right.$, i.e., $M$ is $M_{2 \mathrm{D}, \mathrm{bs}, \text { th }}$ or $\left.M_{3 \mathrm{D}, \mathrm{th}}\right)$.

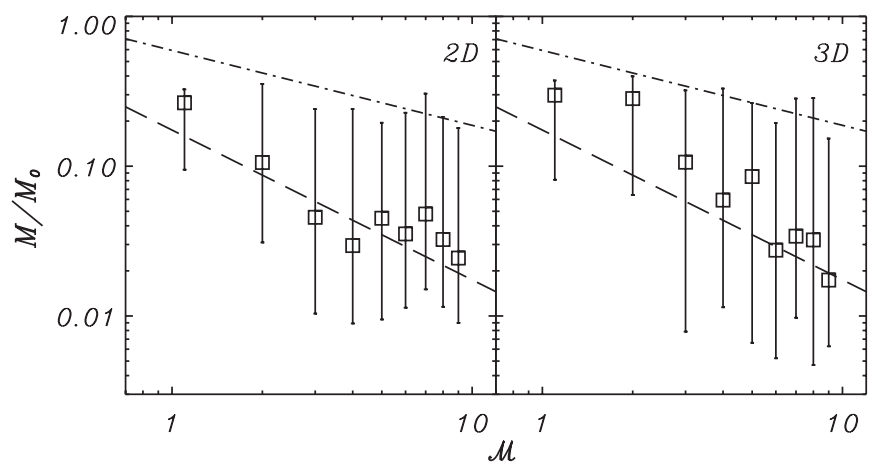

Figure 14. Median GRID-core mass $M_{2 \mathrm{D}, \text { bs }}$ and $M_{3 \mathrm{D}}$, as shown in Figure 12, but for high-amplitude initial perturbations. The median masses are slightly smaller than for low-amplitude initial perturbations, but follow a similar trend. 


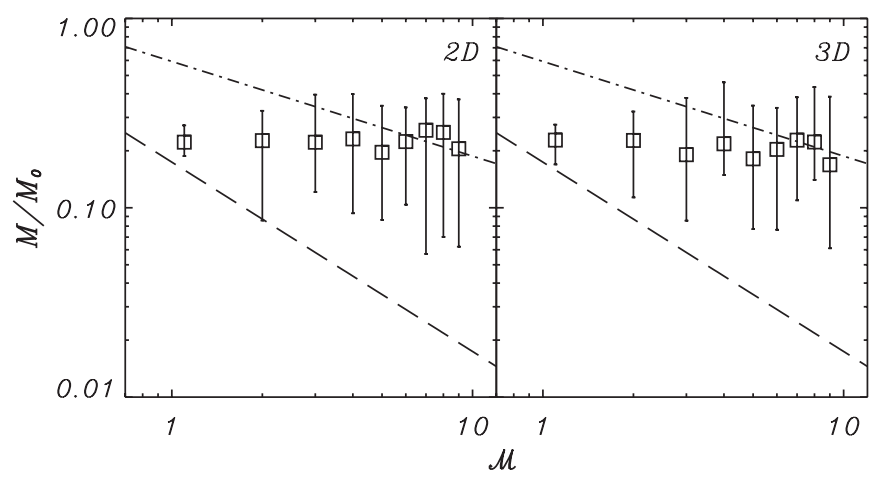

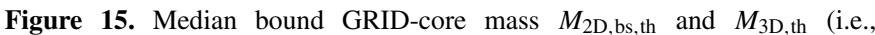
$\left.E_{\mathrm{th}}+E_{g}<0\right)$ as in Figure 13, but for high-amplitude initial perturbations.

Figures 12 and 14). These median masses generally lie above the values predicted from Equations (7), (19), and (22) $\left(M \propto \mathcal{M}^{-1}\right)$ at late stages and below the values predicted from Equation (28) $\left(M \propto \mathcal{M}^{-1 / 2}\right)$ at early stages. The median GRID-core masses for high-amplitude perturbations are slightly smaller than those for low-amplitude perturbations, and the range of core masses for a given Mach number is larger. This reflects the fact that the percentage of small cores is higher when the perturbation amplitudes are higher. GRID-cores are identified based on the gravitational potential, and this potential reflects density structure, which arises from both turbulent and gravitational processes. Even without gravity, smaller-scale masses would be expected in the higher- $\mathcal{M}$ models because of their high turbulent amplitudes. For our simulations, the input perturbation amplitude at scale $l$ is $\delta v_{1 \mathrm{D}}(l)=\left(l / L_{J}\right)^{1 / 2}(\mathcal{M} / 3)^{1 / 2} c_{s}$ at $100 \%$ amplitude of perturbation (cf. Equation (43)). Structures at scales $l$ for which turbulent perturbations are supersonic will, even in the absence of gravity, be more prominent than those at smaller scale. For our adopted scaling of input perturbations with $\mathcal{M}$, the sonic scale varies as $l_{\text {sonic }} \propto L_{J} / \mathcal{M}$, so that the mass at the sonic scale varies $\propto \Sigma(t) l_{\text {sonic }}^{2}$. With $\Sigma(t) \propto \mathcal{M} t_{\text {coll }}$ and $t_{\text {coll }} \propto \mathcal{M}^{-1 / 2}$ (see Equation (29) and below), this predicts $M_{\text {sonic }} \propto \mathcal{M}^{-3 / 2}$. For later time $t \sim t_{J}$ (comparable to the flow crossing time for a cloud with $\left.\alpha_{\text {vir }}=1-2\right), M_{\text {sonic }} \propto \mathcal{M}^{-1}$. Thus, the sonic mass scale, and hence the mass scale of nonlinear structures induced purely by turbulence, is expected to decline with increasing $\mathcal{M}$.

For bound GRID-cores, the median mass versus $\mathcal{M}$ decreases and then increases, for low-amplitude perturbations (Figure 13), and is nearly flat for high-amplitude perturbations (Figure 15). The high median mass at high $\mathcal{M}$ for bound GRID-cores may be due to a combination of effects, including numerical resolution and nonlinearity. The characteristic scale for self-gravitating perturbations decreases with increasing Mach number (either as $r \propto \mathcal{M}^{-1 / 2}$ for the most-grown core or $r \propto \mathcal{M}^{-1}$ for critical perturbations; see Section 2). At high $\mathcal{M}$, this may approach or fall below the minimum scale $r_{\min }=4$ zones $=0.016 L_{J}$ that we require for the GRID-core radius to be well resolved. Since the post-shock density is $\propto \mathcal{M}^{2}$, the GRID-core mass would then increase at least $\propto \mathcal{M}^{2} r_{\text {min }}^{3}$ at sufficiently high $\mathcal{M}$. In addition, larger-scale, higher-mass regions initially have higher amplitude perturbations than smaller-scale regions, because of the input power spectrum with $\delta v \propto l^{1 / 2}$. If this initial "head start" allows the larger, more massive cores to become highly nonlinear before more rapidly growing smaller-scale cores, the more massive cores will collapse (halting the simulation) before the lower-mass cores become strongly concentrated (with

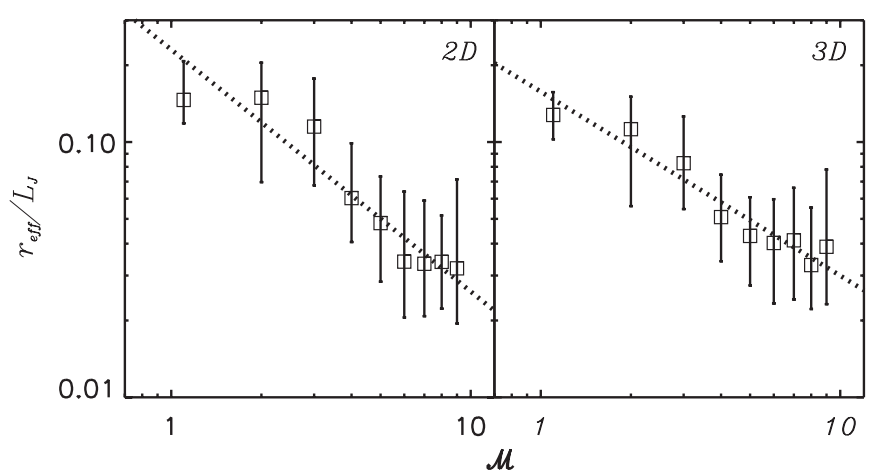

Figure 16. Median GRID-core radius vs. Mach number $\mathcal{M}$ for low-amplitude initial perturbations. Core sizes are defined using the largest closed contours of the gravitational potential in $2 \mathrm{D}\left(\Phi_{2 \mathrm{D}}\right.$, left) and $3 \mathrm{D}(\Phi$, right). Vertical bars indicate quartiles of the distribution. The dotted lines are power-law fits: $r_{\text {eff, } 2 \mathrm{D}, \mathrm{bs}} / L_{J}=0.23_{0.18}^{0.29} \mathcal{M}^{-0.95 \pm 0.13}$ and $r_{\mathrm{eff}, 3 \mathrm{D}} / L_{J}=0.16_{0.14}^{0.18} \mathcal{M}^{-0.72 \pm 0.07}$.

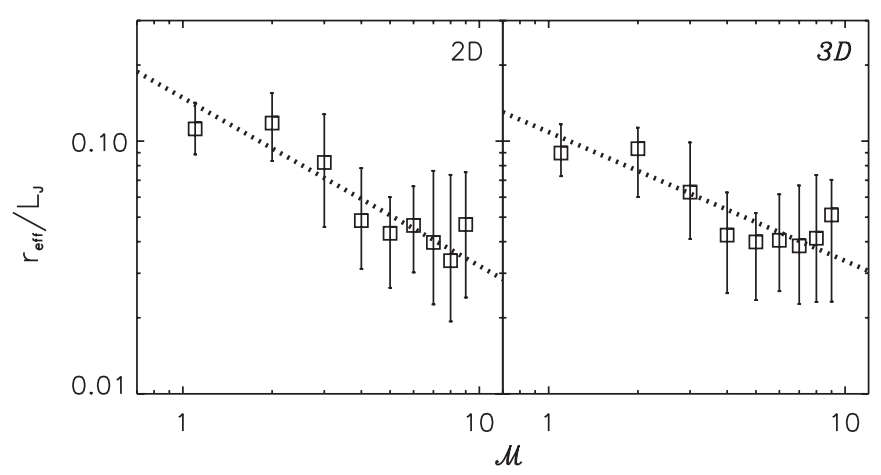

Figure 17. Same as in Figure 16 but for bound GRID-cores $\left(E_{\mathrm{th}}+E_{g}<0\right)$. The power-law fits are $r_{\text {eff,2D,bs,th }} / L_{J}=0.15_{0.12}^{0.18} \mathcal{M}^{-0.67 \pm 0.10}$ and $r_{\text {eff, 3D,th }} / L_{J}=$ $0.11_{0.10}^{0.12} \mathcal{M}^{-0.61 \pm 0.08}$.

$\left.E_{\text {th }}<\left|E_{g}\right|\right)$ internally. With implementation of sink particles such that the simulations need not to be halted when the most evolved core collapses, and $\left|E_{g}\right|$ can grow for low-mass cores, it will be possible to test whether the median mass of bound cores decreases with increasing $\mathcal{M}$, similar to Figures 12 and 14 .

Figure 16 shows the GRID-core radii (as defined in Section 3) versus Mach number, and Figure 17 shows the bound GRIDcore radii versus Mach number; these are for cases with lowamplitude initial perturbations. Overall, the median radii for all GRID-cores and bound GRID-cores decrease toward higher $\mathcal{M}$. This is consistent with expectations: high Mach number yields high post-shock density, and hence a smaller Jeans length; in addition, the higher amplitude of input turbulence at higher $\mathcal{M}$ makes the sonic scale smaller. The prediction for core radius based on turbulence alone would be the sonic scale from Equation (43): $r_{\text {eff }} \propto l_{\text {sonic }} \propto L_{J} / \mathcal{M}$. The first core to collapse is predicted to have $\lambda_{m} \propto \mathcal{M}^{-1 / 2}$ from Equation (30). For latetime fragmentation, the relevant scale is the Jeans length in postshock gas, which varies $\propto \mathcal{M}^{-1}$. For GRID-cores, the slopes are between these values, equal to $-0.95 \pm 0.13$ for $r_{\text {eff, } 2 \mathrm{D}, \text { bs }}$ and $-0.72 \pm 0.07$ for $r_{\mathrm{eff}, 3 \mathrm{D}}$, for low-amplitude initial perturbations. For bound GRID-cores, the power-law fit for median radius as a function of Mach number gives slope $-0.67 \pm 0.10$ and $-0.61 \pm 0.08$ for $2 \mathrm{D}$ and $3 \mathrm{D}$ respectively. These are comparable to the result $\lambda_{m} \propto \mathcal{M}^{-1 / 2}$ from Equation (30). Although the overall slopes are close to -0.5 , we note that the relation flattens at $\mathcal{M} \gtrsim 5$, possibly due to our requirement that the effective radius must exceed four zones, or because the initial power spectrum favors larger cores. 


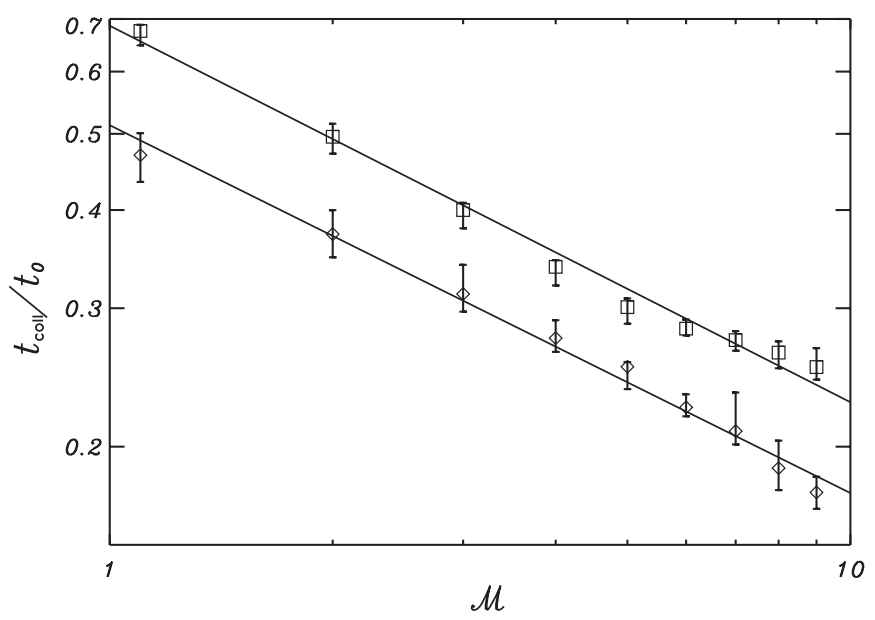

Figure 18. Collapse time of the most evolved core, $t_{\text {coll }}$, vs. inflow Mach number $\mathcal{M}$ for low-amplitude (squares) and high-amplitude (diamonds) initial perturbations. Each value is the median of $t_{\text {coll }}$ for 20 simulations for each $\mathcal{M}$. Vertical bars indicate quartiles of these 20 values of $t_{\text {coll }}$. The solid line least-squares fits are $t_{\text {coll }} / t_{0}=0.69 \mathcal{M}^{-0.48}$ (low amplitude) and $t_{\text {coll }} / t_{0}=$ $0.51 \mathcal{M}^{-0.47}$ (high amplitude). The scaling is comparable to $t_{\text {coll }} \propto \mathcal{M}^{-0.5}$, as predicted by Equation (29). The simulation time unit $t_{0}$, based on the mean inflow density, is given in Equation (6).

Figure 18 shows the median collapse time of the most evolved core vs. Mach number, for both low and high-amplitude initial perturbations. They both follow power laws close to $t_{\text {coll }} \propto \mathcal{M}^{-1 / 2}$, consistent with the timescale (see Equation (29)) predicted for growth of self-gravitating modes up to a given amplification $\Gamma_{\max }$. The coefficients for low-amplitude initial perturbations and high-amplitude initial perturbations are 0.69 and 0.51, respectively, compared to 0.34 from Equation (29) taking $\Gamma_{\max }=1$. With high-amplitude initial perturbations, cores collapse earlier because the seed perturbations need not grow as much. Note that the naive expectation based on the Jeans time, taking $\rho_{\text {post-shock }} \propto \mathcal{M}^{-2}$, would yield a steeper dependence $t \propto \rho_{\text {post-shock }}^{-1 / 2} \propto \mathcal{M}^{-1}$. Based on Figure 18, it is evident that the first cores in higher- $\mathcal{M}$ cases collapse when the layer as a whole is only barely self-gravitating $\left(t_{\text {coll }} / t_{0} \sim\right.$ $0.2-0.3$, compared to $t_{\mathrm{sg}} \approx 0.22 t_{0}$ from Equation (11)), whereas the layer is more strongly self-gravitating at the first collapse for low- $\mathcal{M}$ cases.

The shape of a core can be characterized by the eigenvalues of the moment of inertia tensor $I_{i j} \equiv \int \rho x_{i} x_{j} d^{3} \mathbf{x}$ (e.g., Gammie et al. 2003; Nakamura \& Li 2008). Let $a, b$, and $c$ be the lengths of the principal axes and $a \geqslant b \geqslant c$. Then a prolate core has $b / a=c / a$, and an oblate core has $b / a=1$. We have computed the moment of inertia and aspect ratios for all the cores identified in our simulations. For example, the aspect ratios of the most evolved cores shown in Figures 1 and 3 are $b / a=0.39, c / a=0.25$ for the $\mathcal{M}=1.1$ model and $b / a=0.28, c / a=0.25$ for the $\mathcal{M}=5$ model. They are both (approximately) prolate according to the classification of Gammie et al. (2003).

Figures 19 and 20 show the distribution of core aspect ratios for each $\mathcal{M}$ for low and high-amplitude initial perturbations, respectively. Open circles represent GRID-cores, and dots represent bound GRID-cores. These distributions show a number

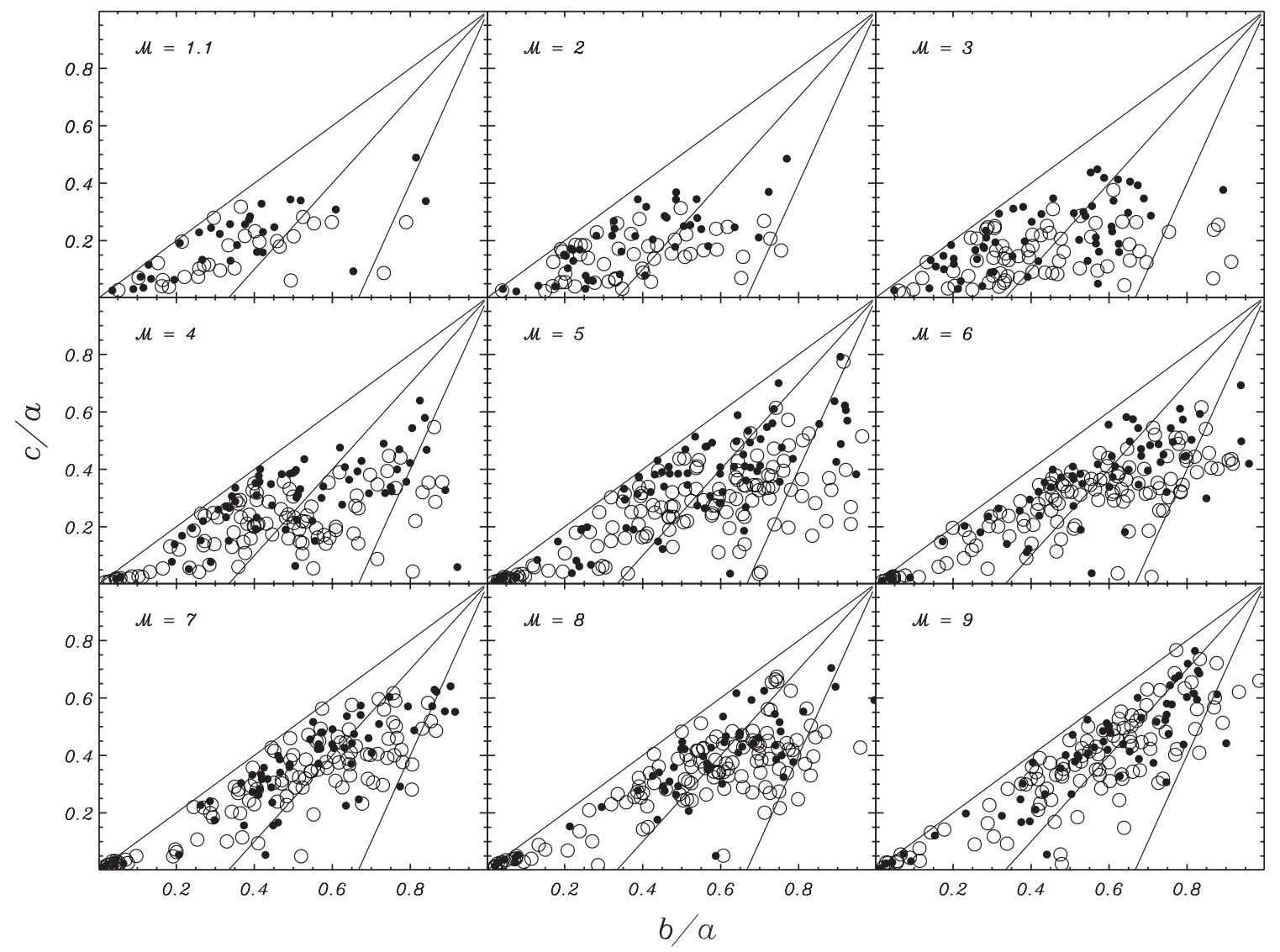

Figure 19. Distribution of three-dimensional core aspect ratio for each Mach number for low-amplitude initial perturbations. Cores lying on $c / a=b / a$ are formally prolate and along $b / a=1$ are formally oblate. We subdivide (see diagonal lines) and classify as follows: approximately prolate (between $c / a=1$ and $c / a=1.5 b / a-0.5$ ), triaxial (between $c / a=1.5 b / a-0.5$ and $c / a=3 b / a-2$ ), and approximately oblate (between $c / a=3 b / a-2$ and $b / a=1$ ). Open circles are GRID-cores defined by the gravitational potential contours alone. Dots are bound GRID-cores, with the additional requirement $E_{\text {th }}+E_{g}<0$. 


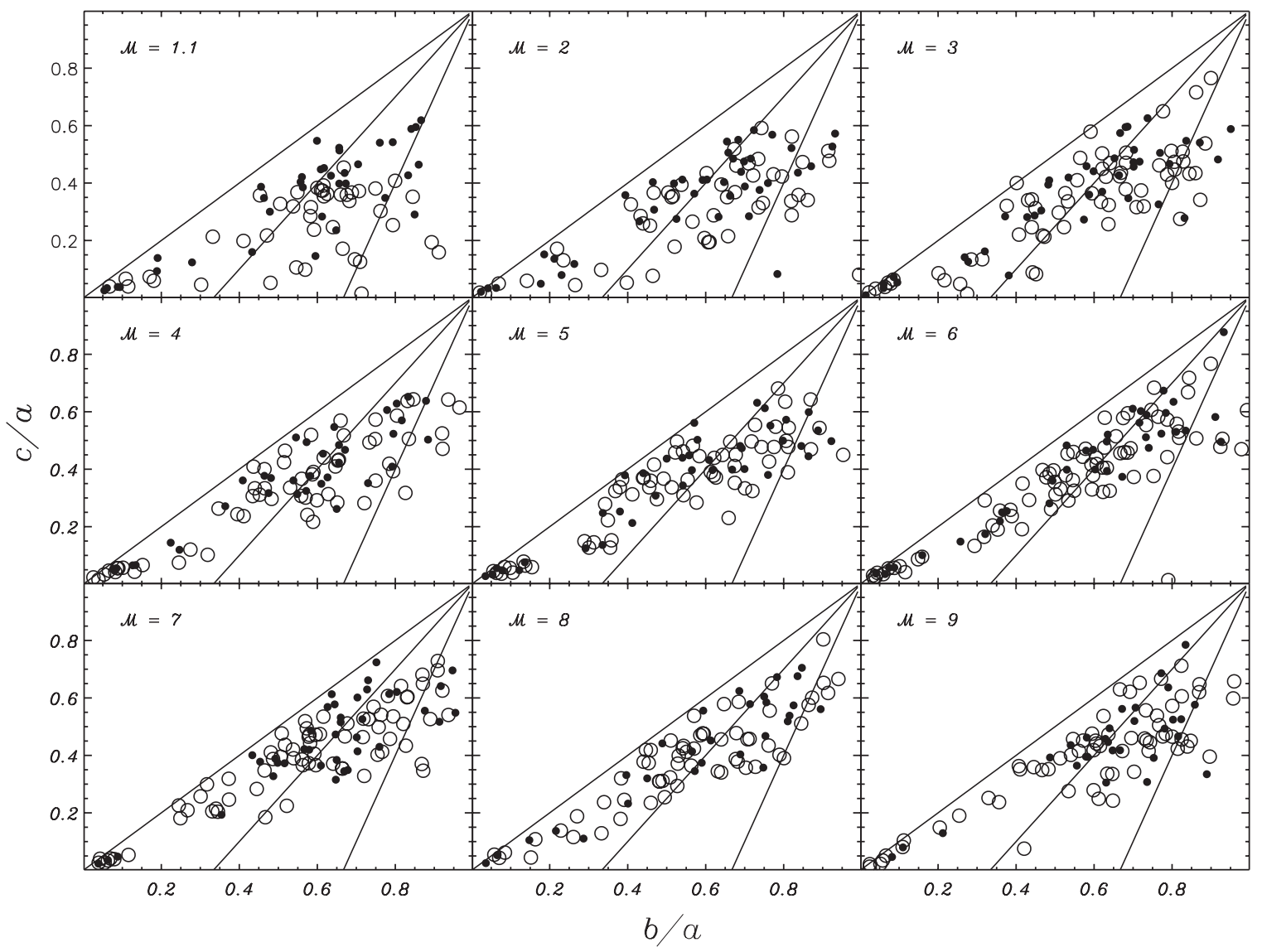

Figure 20. Same as Figure 19 but for high-amplitude initial perturbations.

of interesting features and trends. First, only a small portion of cores are oblate for each $\mathcal{M}$, for both low and high-amplitude perturbations. Second, more oblate-like cores appear when the initial perturbation amplitudes are higher. For low-amplitude perturbations, at $\mathcal{M}=1.1$ and $2, c / a$ and $b / a$ are mostly $\leqslant 0.5$, i.e., approximately prolate. But at larger $\mathcal{M}$ for lowamplitude initial perturbations, and all $\mathcal{M}$ for high-amplitude perturbations, there are many cores in the triaxial and oblate regions. Also, large and massive cores tend to be more prolate. For low-amplitude perturbations, at $\mathcal{M}=1.1$, almost all the cores formed are prolate and no small cores form (compared to high Mach number cases). The reason that the distribution is more oblate for higher amplitude perturbation (large $\mathcal{M}$ for low-amplitude initial perturbations, and all $\mathcal{M}$ for highamplitude initial perturbations) is that more of the cores are at earlier stages of evolution. Figure 1 shows development of cores for $\mathcal{M}=1.1,5$, and 8 . As is particularly clear for the stages shown in the $\mathcal{M}=1.1$ model, structures are more oblate during the core-building stage than during the collapse stage. Cores evolve to become prolate when they collapse because the collapse happens first in the directions perpendicular to the largerscale filaments. For $\mathcal{M}=1.1,2$ models with low-amplitude perturbations, only large cores form and they have evolved to the collapse stage and become prolate. Models with higher amplitude perturbations have a greater percentage of small cores that have not yet collapsed.

We can also examine the relationship between core structure and kinematics in our simulations. Figure 21 shows the projected density field, velocity field and the velocity dispersion field along the line of sight for the $\mathcal{M}=5$ model shown in Figure 6. We "view" the simulation at angles $0^{\circ}, 30^{\circ}$, and $60^{\circ}$ with respect to the $z$-axis, tilting toward the $x$-axis. The white contours mark the regions identified as GRID-cores, and the orange contours mark the bound GRID-cores. The projected density field is smeared as the tilt angle $\theta$ increases. Since $\left\langle v_{\text {los }}\right\rangle=\left\langle v_{x}\right\rangle \sin (\theta)+\left\langle v_{z}\right\rangle \cos (\theta)$, with $\left\langle v_{z}\right\rangle=0$ and the contribution from $\left\langle v_{x}\right\rangle$ small at $\theta$ small, no obvious pattern is seen for $\left\langle v_{\text {los }}\right\rangle$ at $\theta=0^{\circ}$ and $30^{\circ}$. At $\theta=60^{\circ}$, when the $\left\langle v_{x}\right\rangle$ contribution becomes larger, converging-flow patterns similar to those seen in Figure 2 become apparent, especially surrounding the diagonal line of small cores. As previously discussed, converging flows in the $x-y$ plane create this highdensity filament, which then fragments into small cores.

As Figure 21 shows, the dispersions of the line-of-sight velocity of high-density regions are generally subsonic, and are even smaller in the cores. Velocity dispersions are low in high-density regions for two reasons. First, if filaments lie between supersonic converging flows in the $x-y$ plane, then postshock velocities within the filaments will be subsonic. Second, weighting by density picks out regions that are physically small along the line of sight. The increase of linewidth with size means that if a region is smaller than its surroundings along the line of sight, then the linewidth will be smaller than that of its surroundings. Thus, from a combination of low post-shock velocities (in the $x-y$ plane), and spatially limited scale (in the $z$-direction), $\sigma_{\text {los }}$ is low in filaments and lower in cores, as seen in Figure 21.

\section{SUMMARY AND DISCUSSION}

Stars form in GMCs pervaded by supersonic turbulence, and core formation theory must take these supersonic turbulent 

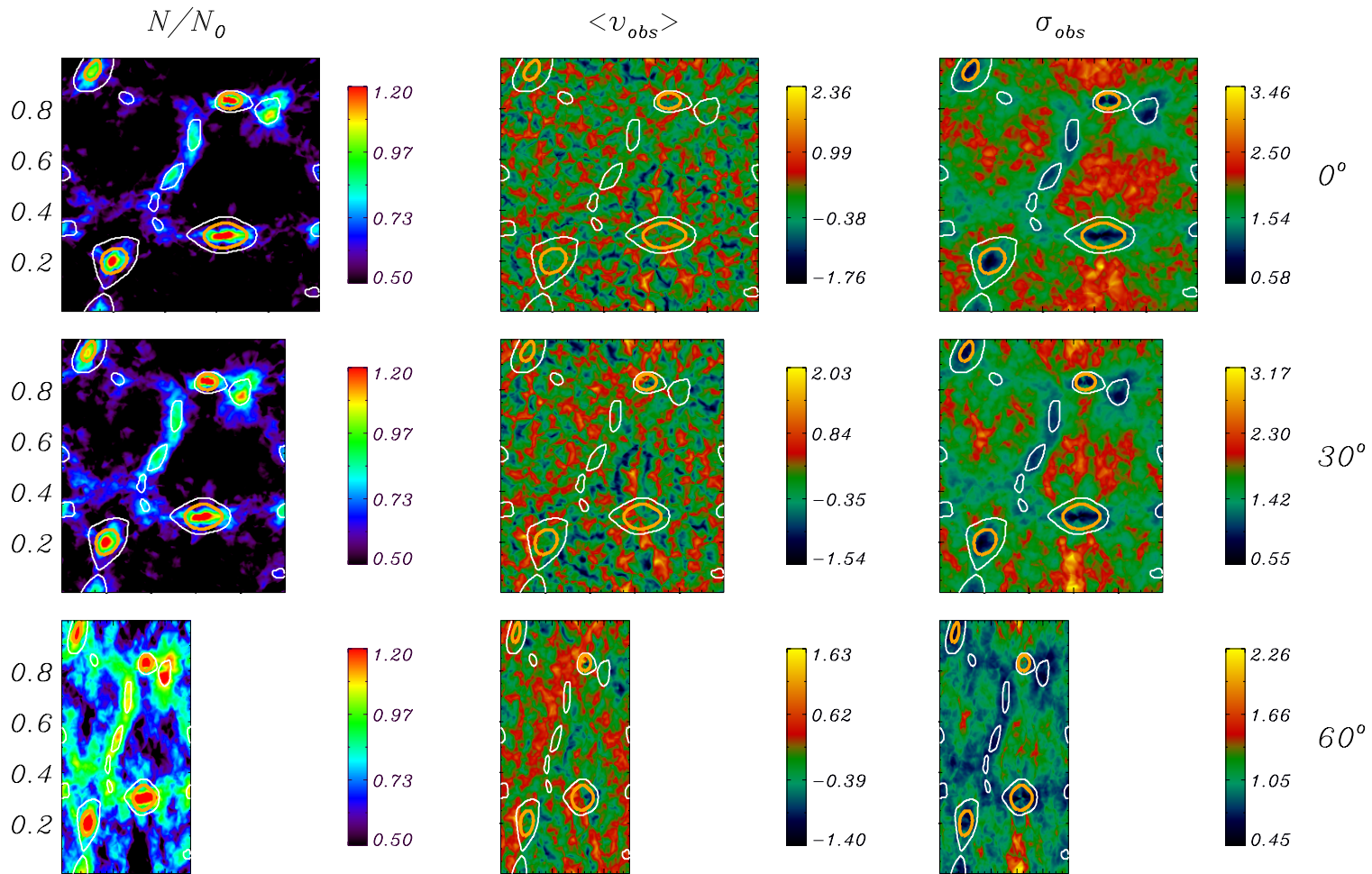

$60^{\circ}$

Figure 21. Observations of one of the $\mathcal{M}=5$ models shown in Figure 6 from different angles. The first column shows the surface density (color scale $\left.\log \Sigma / \Sigma_{0}\right)$; the second column shows the line-of-sight velocity and the third column shows the dispersion of the line-of-sight velocity (linear color scale, in units of $c_{s}$ ). The three rows from top to bottom show the observed fields for $\theta_{\text {tilt }}=0^{\circ}, 30^{\circ}$, and $60^{\circ}$, respectively. The white curves are the GRID-cores, and the orange curves are the bound GRID-cores. Note that core regions have low internal velocity dispersions.

(A color version of this figure is available in the online journal.)

flows into account. In this work, we explore the physics of core formation in a dynamic environment, focusing on postshock layers generated by collisions of supersonic flows. The framework we adopt-three-dimensional planar converging flows containing multi-scale turbulence-enables us to analyze the internal structure and kinematics of cores, and to investigate the relation between core properties and the inflow Mach number $\mathcal{M}$. We consider a range $\mathcal{M}=1.1-9$, and conduct 180 simulations with different realizations of the initial turbulent power spectrum, in order to obtain a sizable statistical sample. In addition to core masses and sizes, we measure aspect ratios. To define cores, we introduce a new method based on the gravitational potential, and compare properties of cores identified using $\Phi$ (from the volume density) and $\Phi_{2 \mathrm{D}}$ (from the plane-of-sky projected surface density).

Unlike previous studies of core evolution that begin with pre-existing cores, the present models include formation stages. Our initial density is uniform everywhere, and cores grow, via self-gravity, from turbulence-induced perturbations within the post-shock layer; when the Mach number is high, initial growth of density perturbations is aided by shock-driven hydrodynamic instabilities. Based on a set of spherically symmetric numerical simulations, Gong \& Ostriker (2009) proposed four stages for core evolution in dynamic environments: core building, core collapse, envelope infall, and late accretion. The key features during core building and collapse described in Gong \& Ostriker (2009) are verified here, for more realistic geometry. As the supersonic flows converge in a plane, two reversed shocks propagate outward. With its high mean density, the stagnation layer between these two shock fronts becomes an incubator for self-gravitating cores. When these cores become sufficient stratified, they collapse. We halt the simulations at the instant of singularity formation in the most evolved core, because the time step becomes very short.

Based on the analysis of our simulations, our chief conclusions are as follows:

1. Cores with realistic properties are able to form in post-shock dense layers within turbulent GMCs. For core building to become supercritical, it takes $\sim 10$ times as long as the subsequent "outside-in" collapse stage, which lasts a few $\times 10^{5} \mathrm{yr}$. The duration of the supercritical stage is consistent with observations of prestellar core lifetimes (Ward-Thompson et al. 2007; Enoch et al. 2008; Evans et al. 2009).

2. At the time of singularity formation, the radial density profile within cores approaches the Larson-Penston asymptotic solution $\rho=8.86 c_{s}^{2} /\left(4 \pi G r^{2}\right)$ and the velocity approaches the Larson-Penston limit $-3.28 c_{s}$. This is consistent with previous studies of spherical core collapse (see Section 1 for references). Tilley \& Pudritz (2004) also found that $\rho \propto r^{-2}$ in their most massive cores, for turbulent simulations. As in Gong \& Ostriker (2009), we therefore conclude that the Larson-Penston asymptotic solution is an "attractor" for core collapse, no matter how the collapse is initiated.

3. Prior to collapse, the velocities within dense cores remain subsonic, in spite of the highly supersonic flows that create them. This is true both for the ordered inflow, and for the mean internal velocity dispersion. This result is consistent with observations that most cores have subsonic nonthermal velocity dispersions (Myers 1983; Goodman et al. 1998; Caselli et al. 2002; Tafalla et al. 2004; Kirk et al. 2007; André et al. 2007; Lada et al. 2008). The velocity 
dispersion can increase quite sharply at the edge of the core in our models (see Figure 21), intriguingly similar to a sharp transition seen in $\mathrm{NH}_{3}$ observations by Pineda et al. (2010) for the B5 core in Perseus. From some orientations, velocity dispersions in filaments containing cores may also be lower than in the surrounding gas (cf. Figure 21).

4. At sub-parsec scales, turbulent velocity perturbations (whether super- or subsonic) induce density perturbations that can grow strongly if the density is high enough for self-gravity to be important. In post-shock layers, turbulence and self-gravity collect gas into long, thin filamentary structures at the same time as the highest density regions within the filaments grow to become centrally condensed cores. These filamentary structures containing embedded cores are similar to the structures in the Aquila rift and Polaris Flare clouds observed by Herschel (André et al. 2010; Men'shchikov et al. 2010).

5. Using the gravitational potential to identify cores is advantageous because it enables a core definition based on dynamical principles. For numerical simulations, the gravitational potential may be computed from the volume density (yielding $\Phi$ ) or from the projected surface density (yielding $\left.\Phi_{2 \mathrm{D}}\right)$. We show for our models that cores defined using $\Phi$ and $\Phi_{2 D}$ are nearly the same, both for GRID-cores (defined by the largest closed potential isosurfaces) and bound GRID-cores (which additionally require $E_{\text {th }}+E_{g}<0$ ). Since $\Phi_{2 \mathrm{D}}$ can be computed for observed clouds, using potential contours offers a promising new core identification method for application to high-resolution molecular cloud maps. IDL code implementing our GRID-core algorithm, suitable for application to observed data, is available from the authors.

6. We find that the range of core masses that form increases as the Mach number $\mathcal{M}$ increases. Physically, this is because a larger range of spatial scales has significant perturbations when the turbulence amplitude is higher, and because the minimum mass to be gravitationally unstable decreases as the density in the shocked layer increases. Basu et al. (2009) also found broader mass distributions when the turbulent amplitude is increased. At high Mach number, GRIDcore masses range between $\sim 10^{-3}-1 M_{J}$, corresponding to $\sim 0.05-50 M_{\odot}$ for typical GMC conditions.

7. Analytical arguments (see Section 2) suggest that the first core to collapse will have mass $M \propto \mathcal{M}^{-1 / 2}$, and that at late times, the minimum mass core will vary as $M \propto \mathcal{M}^{-1}$. Our numerical results for median core masses as a function of $\mathcal{M}$ lie between these two relations. When the core definition includes the condition that $E_{\mathrm{th}}+E_{g}<0$, the median mass increases at the largest Mach number. This may be due to the nonlinear "head start" of massive cores, such that lowermass cores have not yet become concentrated when the first core collapses (and the simulation is stopped).

8. Analytical arguments (see Section 2) suggest that the effective core radius will decline with increasing Mach number, with powers between $r_{\text {eff }} \propto \mathcal{M}^{-1 / 2}$ and $r_{\text {eff }} \propto$ $\mathcal{M}^{-1}$. Our numerical results show a decrease of $r_{\text {eff }}$ with $\mathcal{M}$ in this range. For bound GRID-cores $\left(E_{\mathrm{th}}+E_{g}<0\right)$, the relation is shallower than for GRID-cores defined by gravitational potential alone.

9. The time for the first core to collapse in our simulations depends on Mach number, with $t_{\text {coll }} \propto \mathcal{M}^{-1 / 2}$, and a slightly smaller coefficient for high-amplitude initial perturbations (see Figure 18). This scaling is consistent with analytic pre- dictions for gravitational instability in a shocked converging flow (see Equation (29)). For high $\mathcal{M}$, as is observed in GMCs, the first cores could collapse within a few Myr of cloud formation. For high $\mathcal{M}$, the first cores collapse when the shocked layer containing them is only barely selfgravitating; this suggests that collections of stars can begin to form individually before they collapse together to create a cluster.

10. A very small portion of cores are oblate, while most cores are prolate or triaxial. Large cores are preferentially prolate. The triaxiality of most cores is consistent with previous results from turbulent hydrodynamic and MHD simulations (Gammie et al. 2003; Li et al. 2004; Nakamura \& Li 2008; Offner et al. 2008). We also find that core shapes change as they evolve, from more oblate during early stages to more prolate during collapse. For high initial perturbation amplitudes, the distributions have a higher proportion of oblate cores because small cores are less evolved (at the time the first core collapses), compared to those in models with low initial perturbation amplitudes.

As noted above, the current models have provided evidence that the masses of cores that form depend not just on the mean Jeans mass in a cloud, but also on the cloud's level of internal turbulence at large scales, $\sigma_{v}$. Equations (22) and (23) suggest that at late times, the characteristic core mass will follow $M_{c} \propto \sigma_{v}^{-1} \rho_{0}^{-1 / 2} T^{2}$, where $\rho_{0}$ is the mean density in the cloud. For the current simulations, however, we halt at the instant when the most evolved core collapses (because the time step becomes very short). This limits the condensation of small cores; they are present, but not yet strongly bound. In order to fully test the dependence of $M_{c}$ on cloud parameters, it is necessary to implement sink particles (e.g., Krumholz et al. 2004; Federrath et al. 2010) so that the simulation can run until all the "eligible" cores in the post-shock region have had the opportunity to collapse. Including sink particles, as well as studying shocked converging flows within larger turbulent clouds via mesh-refined simulations, represent important avenues for future research.

We are grateful to Lee Mundy and Alyssa Goodman for stimulating conversations, and to the referee for a helpful report. This work was supported by grants NNX09AG04G and NNX10AF60G from NASA.

\section{REFERENCES}

Alves, J. F., Lada, C. J., \& Lada, E. A. 2001, Nature, 409, 159

Alves, J., Lombardi, M., \& Lada, C. J. 2007, A\&A, 462, L17

André, P., Basu, S., \& Inutsuka, S.-I. 2008, in Structure Formation in Astrophysics, ed. G. Chabrier (Cambridge: Cambridge Univ. Press), 254

André, P., Belloche, A., Motte, F., \& Peretto, N. 2007, A\&A, 472, 519

André, P., et al. 2010, A\&A, 518, L102

Bacmann, A., André, P., Puget, J.-L., Abergel, A., Bontemps, S., \& Ward-Thompson, D. 2000, A\&A, 361, 555

Ballesteros-Paredes, J., Klessen, R. S., Mac Low, M.-M., \& Vazquez-Semadeni, E. 2007, in Protostars and Planets V, ed. B. Reipurth, D. Jewitt, \& K. Keil (Tucson, AZ: Univ. Arizona Press), 63

Banerjee, R., Vázquez-Semadeni, E., Hennebelle, P., \& Klessen, R. S. 2009, MNRAS, 398, 1082

Basu, S., Ciolek, G. E., Dapp, W. B., \& Wurster, J. 2009, New Astron., 14, 483 Bergin, E. A., \& Tafalla, M. 2007, ARA\&A, 45, 339

Beuther, H., \& Schilke, P. 2004, Science, 303, 1167

Blitz, L., Fukui, Y., Kawamura, A., Leroy, A., Mizuno, N., \& Rosolowsky, E. 2007, in Protostars and Planets V, ed. B. Reipurth, D. Jewitt, \& K. Keil (Tucson, AZ: Univ. Arizona Press), 81

Bodenheimer, P., \& Sweigart, A. 1968, ApJ, 152, 515

Bonnell, I. A., Bate, M. R., \& Vine, S. G. 2003, MNRAS, 343, 413

Bonnell, I. A., Clarke, C. J., \& Bate, M. R. 2006, MNRAS, 368, 1296 
Bonnor, W. B. 1956, MNRAS, 116, 351

Burkert, A., \& Alves, J. 2009, ApJ, 695, 1308

Caselli, P., Benson, P. J., Myers, P. C., \& Tafalla, M. 2002, ApJ, 572, 238

Chabrier, G. 2005, in The Initial Mass Function 50 Years Later, ed. E. Corbelli, F. Palle, \& H. Zinnecker (ASSL Vol. 327; Dordrecht: Springer), 41

Ciolek, G. E., \& Basu, S. 2001, ApJ, 547, 272

Ciolek, G. E., \& Mouschovias, T. C. 1994, ApJ, 425, 142

Clark, P. C., Bonnell, I. A., \& Klessen, R. S. 2008, MNRAS, 386, 3

Dapp, W. B., \& Basu, S. 2009, MNRAS, 395, 1092

di Francesco, J., Evans, N. J., II, Caselli, P., Myers, P. C., Shirley, Y., Aikawa, Y., \& Tafalla, M. 2007, in Protostars and Planets V, ed. B. Reipurth, D. Jewitt, \& K. Keil (Tucson, AZ: Univ. Arizona Press), 17

Di Francesco, J., Myers, P. C., Wilner, D. J., Ohashi, N., \& Mardones, D. 2001, ApJ, 562, 770

Ebert, R. 1955, Zs. Ap., 37, 217

Elmegreen, B. G., \& Elmegreen, D. M. 1978, ApJ, 220, 1051

Enoch, M. L., Evans, N. J., II, Sargent, A. I., Glenn, J., Rosolowsky, E., \& Myers, P. 2008, ApJ, 684, 1240

Enoch, M. L., et al. 2006, ApJ, 638, 293

Evans, N. J., et al. 2009, ApJS, 181, 321

Federrath, C., Banerjee, R., Clark, P. C., \& Klessen, R. S. 2010, ApJ, 713, 269

Foster, P. N., \& Chevalier, R. A. 1993, ApJ, 416, 303

Gammie, C. F., Lin, Y.-T., Stone, J. M., \& Ostriker, E. C. 2003, ApJ, 592, 203

Gardiner, T. A., \& Stone, J. M. 2005, J. Comput. Phys., 205, 509

Gardiner, T. A., \& Stone, J. M. 2008, J. Comput. Phys., 227, 4123

Gómez, G. C., Vázquez-Semadeni, E., Shadmehri, M., \& Ballesteros-Paredes, J. 2007, ApJ, 669, 1042

Gong, H., \& Ostriker, E. C. 2009, ApJ, 699, 230

Goodman, A. A., Barranco, J. A., Wilner, D. J., \& Heyer, M. H. 1998, ApJ, 504, 223

Gregersen, E. M., Evans, N. J., II, Zhou, S., \& Choi, M. 1997, ApJ, 484, 256

Heitsch, F., Hartmann, L. W., Slyz, A. D., Devriendt, J. E. G., \& Burkert, A. 2008, ApJ, 674, 316

Heitsch, F., Slyz, A. D., Devriendt, J. E. G., Hartmann, L. W., \& Burkert, A. 2007, ApJ, 665, 445

Hennebelle, P., \& Chabrier, G. 2008, ApJ, 684, 395

Hennebelle, P., Whitworth, A. P., Gladwin, P. P., \& Andre, Ph. 2003, MNRAS, 340,870

Heyer, M. H., \& Brunt, C. M. 2004, ApJ, 615, L45

Heyer, M., Krawczyk, C., Duval, J., \& Jackson, J. M. 2009, ApJ, 699, 1092

Hunter, C. 1977, ApJ, 218, 834

Ikeda, N., \& Kitamura, Y. 2009, ApJ, 705, L95

Ikeda, N., Kitamura, Y., \& Sunada, K. 2009, ApJ, 691, 1560

Ikeda, N., Sunada, K., \& Kitamura, Y. 2007, ApJ, 665, 1194

Iwasaki, K., \& Tsuribe, T. 2008, PASJ, 60, 125

Johnstone, D., Fich, M., Mitchell, G. F., \& Moriarty-Schieven, G. 2001, ApJ, 559,307

Johnstone, D., Wilson, C. D., Moriarty-Schieven, G., Joncas, G., Smith, G., Gregersen, E., \& Fich, M. 2000, ApJ, 545, 327

Kandori, R., et al. 2005, AJ, 130, 2166

Kim, W.-T., Ostriker, E. C., \& Stone, J. M. 2002, ApJ, 581, 1080

Kirk, H., Johnstone, D., \& Tafalla, M. 2007, ApJ, 668, 1042

Kirk, J. M., Ward-Thompson, D., \& André, P. 2005, MNRAS, 360, 1506

Klessen, R. S. 2001, ApJ, 556, 837

Könyves, V., et al. 2010, A\&A, 518, L106

Koyama, H., \& Ostriker, E. C. 2009, ApJ, 693, 1316

Kroupa, P. 2001, MNRAS, 322, 231

Krumholz, M. R., McKee, C. F., \& Klein, R. I. 2004, ApJ, 611, 399

Kudoh, T., \& Basu, S. 2008, ApJ, 679, L97

Lada, C. J., Muench, A. A., Rathborne, J., Alves, J. F., \& Lombardi, M. 2008, ApJ, 672, 410

Larson, R. B. 1969, MNRAS, 145, 271

Larson, R. B. 1981, MNRAS, 194, 809

Lee, C. W., \& Myers, P. C. 1999, ApJS, 123, 233

Lee, C. W., Myers, P. C., \& Tafalla, M. 2001, ApJS, 136, 703

Li, P. S., Norman, M. L., Mac Low, M.-M., \& Heitsch, F. 2004, ApJ, 605, 800
Lubow, S. H., \& Pringle, J. E. 1993, MNRAS, 263, 701

McKee, C. F., \& Ostriker, E. C. 2007, ARA\&A, 45, 565

Men'shchikov, A., et al. 2010, A\&A, 518, L103

Mestel, L., \& Spitzer, L., Jr. 1956, MNRAS, 116, 503

Motoyama, K., \& Yoshida, T. 2003, MNRAS, 344, 461

Motte, F., Andre, P., \& Neri, R. 1998, A\&A, 336, 150

Motte, F., André, P., Ward-Thompson, D., \& Bontemps, S. 2001, A\&A, 372, L41

Mouschovias, T. C. 1987, in Physical Processes in Interstellar Clouds, ed. G. E. Mofrill \& M. Scholer (Dordrecht: Reidel), 453

Mouschovias, T. C., \& Ciolek, G. E. 1999, in NATO ASIC Proc.540, The Origin of Stars and Planetary Systems, ed. C. J. Lada \& N. D. Kylafis (Dordrecht: Kluwer), 305

Mouschovias, T. C., \& Spitzer, L., Jr. 1976, ApJ, 210, 326

Myers, P. C. 1983, ApJ, 270, 105

Nakamura, F., \& Li, Z.-Y. 2008, ApJ, 687, 354

Nakano, T., \& Nakamura, T. 1978, PASJ, 30, 671

Nutter, D., \& Ward-Thompson, D. 2007, MNRAS, 374, 1413

Offner, S. S. R., Klein, R. I., \& McKee, C. F. 2008, ApJ, 686, 1174

Ogino, S., Tomisaka, K., \& Nakamura, F. 1999, PASJ, 51, 637

Onishi, T., Mizuno, A., Kawamura, A., Tachihara, K., \& Fukui, Y. 2002, ApJ, 575,950

Ostriker, E. C., Gammie, C. F., \& Stone, J. M. 1999, ApJ, 513, 259

Padoan, P., Jones, B. J. T., \& Nordlund, A. P. 1997, ApJ, 474, 730

Padoan, P., \& Nordlund, Å. 2002, ApJ, 576, 870

Padoan, P., \& Nordlund, A. 2004, ApJ, 617, 559

Padoan, P., Nordlund, Å., Kritsuk, A. G., Norman, M. L., \& Li, P. S. 2007, ApJ, 661,972

Pavlovski, G., Smith, M. D., \& Mac Low, M.-M. 2006, MNRAS, 368, 943

Penston, M. V. 1969, MNRAS, 144, 425

Pineda, J. E., Goodman, A. A., Arce, H. G., Caselli, P., Foster, J. B., Myers, P. C., \& Rosolowsky, E. W. 2010, ApJ, 712, L116

Pineda, J. E., Rosolowsky, E. W., \& Goodman, A. A. 2009, ApJ, 699, L134

Rathborne, J. M., Lada, C. J., Muench, A. A., Alves, J. F., Kainulainen, J., \& Lombardi, M. 2009, ApJ, 699, 742

Reid, M. A., \& Wilson, C. D. 2005, ApJ, 625, 891

Reid, M. A., \& Wilson, C. D. 2006, ApJ, 644, 990

Shirley, Y. L., Evans, N. J., II, Rawlings, J. M. C., \& Gregersen, E. M. 2000, ApJS, 131, 249

Shu, F. H. 1977, ApJ, 214, 488

Shu, F. H., Adams, F. C., \& Lizano, S. 1987, ARA\&A, 25, 23

Simpson, R. J., Nutter, D., \& Ward-Thompson, D. 2008, MNRAS, 391, 205

Smith, R. J., Clark, P. C., \& Bonnell, I. A. 2009, MNRAS, 396, 830

Sohn, J., Lee, C. W., Park, Y.-S., Lee, H. M., Myers, P. C., \& Lee, Y. 2007, ApJ, 664,928

Solomon, P. M., Rivolo, A. R., Barrett, J., \& Yahil, A. 1987, ApJ, 319, 730

Stanke, T., Smith, M. D., Gredel, R., \& Khanzadyan, T. 2006, A\&A, 447, 609

Stone, J. M., \& Gardiner, T. 2009, New Astron., 14, 139

Stone, J. M., Gardiner, T. A., Teuben, P., Hawley, J. F., \& Simon, J. B. 2008, ApJS, 178, 137

Tafalla, M., Myers, P. C., Caselli, P., \& Walmsley, C. M. 2004, A\&A, 416, 191

Testi, L., \& Sargent, A. I. 1998, ApJ, 508, L91

Tilley, D. A., \& Pudritz, R. E. 2004, MNRAS, 353, 769

Toro, E. F. 1999, Riemann Solvers and Numerical Methods for Fluid Dynamics (Berlin: Springer)

Troland, T. H., \& Crutcher, R. M. 2008, ApJ, 680, 457

Vazquez-Semadeni, E. 1994, ApJ, 423, 681

Vishniac, E. T. 1994, ApJ, 428, 186

Vorobyov, E. I., \& Basu, S. 2005, MNRAS, 360, 675

Ward-Thompson, D., André, P., Crutcher, R., Johnstone, D., Onishi, T., \& Wilson, C. 2007, in Protostars and Planets V, ed. B. Reipurth, D. Jewitt, \& K. Keil (Tucson, AZ: Univ. Arizona Press), 33

Whitworth, A. P., Bhattal, A. S., Chapman, S. J., Disney, M. J., \& Turner, J. A. 1994, A\&A, 290, 421

Williams, J. P., de Geus, E. J., \& Blitz, L. 1994, ApJ, 428, 693 